\title{
Aggregation Latency-Energy Tradeoff in Wireless Sensor Networks with Successive Interference Cancellation
}

\author{
Hongxing Li, Chuan Wu, Dongxiao Yu, Qiang-Sheng Hua and Francis C.M. Lau \\ Department of Computer Science, The University of Hong Kong, Hong Kong \\ Email: $\{$ hxli, cwu, dxyu, qshua, fcmlau\}@cs.hku.hk
}

\begin{abstract}
Minimizing latency and energy consumption is the prime objective of the design of data aggregation in battery-powered wireless networks. A tradeoff exists between the aggregation latency and the energy consumption, which has been widely studied under the protocol interference model. There has been however no investigation of the tradeoff under the physical interference model which is known to capture more accurately the characteristics of wireless interferences. When coupled with the technique of successive interference cancellation, by which a receiver may recover signals from multiple simultaneous senders, the model can lead to much reduced latency but increased energy usage. In this paper, we investigate the latency-energy tradeoff for data aggregation in wireless sensor networks under the physical interference model and using successive interference cancellation. We present theoretical lower bounds on both latency and energy as well as their tradeoff, and give an efficient approximation algorithm that can achieve the asymptotical optimum in both aggregation latency and latency-energy tradeoff. We show that our algorithm can significantly reduce the aggregation latency, for which the energy consumption is kept at its lowest possible level.
\end{abstract}

Index Terms-Data aggregation, Latency-energy tradeoff, Wireless sensor network, Successive interference cancellation.

\section{INTRODUCTION}

Wireless sensor networks have been extensively exploited for many environment monitoring applications in recent years. One of the core functions in these networks is data aggregation, which is to collect data from the wireless sensor nodes to deliver to a sink node. Typically, data aggregation is initiated by the sink using some SQL-like queries, such as "to find the highest temperature in the region". Messages generated at individual sensors carrying temperature data, are first aggregated and processed at some relay sensors, e.g., to derive the local maximum temperature; the locally processed results are further aggregated, and so on, until the final result reaches the sink. Besides the $\max$ function, other functions such as min, sum, count, and average can all be effectively implemented using data aggregation.

As the sensed data typically has a limited duration of validity, a fundamental requirement is that the total aggregation time, measured in time units and also referred to as the aggregation latency, must be minimized [1]-[3]. Additionally, the sensor nodes have to observe the hard constraint imposed by battery power and must strive for low energy consumption in each run of the data aggregation. Obviously, there exists some kind of tradeoff between aggregation latency and energy consumption (the latencyenergy tradeoff) in wireless sensor data aggregation [4]-[6].

There have been some efforts to derive latency-energy tradeoff theoretically [7] as well as practical algorithms [4][6], which are all based on the protocol interference model (or equivalently the pair-wise interference model). Under the protocol interference model, the transmission range and interference range of a node are simplified to two disks with radii $r_{t}$ and $r_{i}\left(r_{i} \geq r_{t}\right)$, respectively. A transmission is successful if and only if the receiver lies within the transmis- sion range of the sender and outside the interference range of any other concurrent sender. There has been however no prior study that is based on the physical interference model (or the cumulative interference model) which has been shown to be able to more accurately characterize the wireless interferences than the protocol interference model [8]-[10]. Designs based on the physical interference model can lead to increased network capacity. Under the physical interference model, the cumulative interference from all concurrent transmissions, e.g. the $\sum_{e_{j} \in \Lambda_{i}} P_{j} / d_{j i}^{\alpha}$ part in Eqn. (1), is taken into consideration at each receiver. A transmission along link $e_{i}$ is successful if the Signal-toInterference-plus-Noise-Ratio (SINR) at its receiver is above a certain threshold:

$$
\frac{P_{i} / d_{i i}^{\alpha}}{N_{0}+\sum_{e_{j} \in \Lambda_{i}} P_{j} / d_{j i}^{\alpha}} \geq \beta .
$$

Here $\Lambda_{i}$ denotes the set of links that transmit simultaneously with $e_{i} . P_{i}$ and $P_{j}$ denote the transmission powers at the transmitter of link $e_{i}$ and that of link $e_{j}$, respectively. $d_{i i}$ $\left(d_{j i}\right)$ is the distance between the transmitter of link $e_{i}\left(e_{j}\right)$ and the receiver of link $e_{i}$. Fig. 1 explains these distances graphically. $\alpha$ is the path loss ratio which has a typical value of between 2 to $6 . N_{0}$ is the ambient noise power. $\beta$ is a positive constant as the SINR threshold for a successful transmission [3], [11].

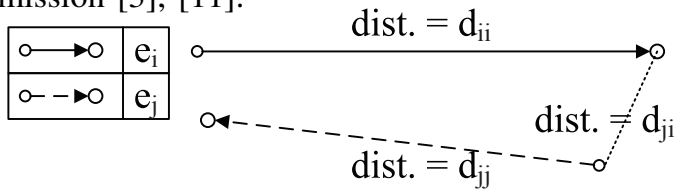

Fig. 1: An illustration of distances with two transmission links: $e_{i}$ and $e_{j}$.

With the physical interference model, a receiver can only successfully recover one signal from one sender in each 
time slot, among possibly several or many simultaneous transmissions. In recent years, it has been shown that, by applying Multi-Packet Reception (MPR) techniques [12], it is possible to break this one-timeslot-one-sender barrier to let a receiver recover multiple individual signals from the mixed signal coming from multiple simultaneous senders. Successive Interference Cancellation (SIC), one subcategory of $M P R$, has been demonstrated practical by experimental study [13] implemented for the IEEE 802.15.4 [14] (ZigBee) physical layer (a common physical-layer standard for sensor networks and other wireless personal area networks). The idea of SIC is to repeatedly identify the strongest signal and then remove (cancel) it from the mixed one using channel estimation, signal regenerating and subtraction [15]. To make it work, an interference cancellation sequence needs to be identified, such that for the $i^{t h}$ signal to be canceled, the following criterion is satisfied:

$$
\frac{P_{i} / d_{i i}^{\alpha}}{N_{0}+\sum_{e_{j} \in \Lambda_{i}-\Gamma_{i}} P_{j} / d_{j i}^{\alpha}+\sum_{e_{k} \in \Gamma_{i}, k \succ i} P_{k} / d_{k i}^{\alpha}} \geq \beta,
$$

where $\Gamma_{i}$ is the set of concurrent transmission links connecting to the same receiver as $e_{i}$ 's, and $k \succ i$ denotes that link $e_{i}$ is canceled before link $e_{k}$. Extra energy is needed to recover the $i^{t h}$ signal if it is not the last canceled one, in order to compensate for the cumulative interference from those links that are later canceled. Meanwhile, extra decoding delay, proportional to the number of canceled signals, is incurred for the entire signal cancellation process [13].

SIC techniques can potentially reduce the aggregation latency in wireless sensor networks significantly, because multiple transmissions can be scheduled in the same time slot while the saved scheduling latency overwhelms the incurred decoding delay (To be compared in Sec. 3.2). Inevitably, the cost is increased energy consumption. To the best of our knowledge, there is no previous study that has tried to characterize the latency-energy tradeoff under SIC. Such characterization is needed in order to accurately gauge the practical benefits of applying SIC in typical wireless applications.

In this paper, we investigate the aggregation latencyenergy tradeoff in wireless sensor networks under the physical interference model with successive interference cancellation. Our contributions are as follows:

$\triangleright$ We prove a theoretical lower bound on the aggregation latency under the physical interference model with SIC: $\Omega\left(\max \left\{D, \log _{X+1} n\right\}\right)$, where $D$ is the network diameter in terms of the number of hops (the maximum of the minimum number of hops between any pair of nodes, when the nodes are transmitting using $P_{M}$ and scheduled without mutual interference), $n$ is the number of nodes, and $X=\left\lfloor\log _{1+\beta} \frac{P_{M}}{N_{0} \beta}+1\right\rfloor$ with $P_{M}$ being the maximum transmission power of any node.

$\triangleright$ We prove a theoretical lower bound, applicable to both the case with and that without SIC, on energy consumption under the physical interference model: $N_{0} \beta \frac{\left(n_{m i s} d_{M}\right)^{\alpha}}{n^{\alpha-1}}$, where $n_{m i s}$ is the size of the maximum independent set with $P_{M}$ (see Definition 1 in Section 4) of the given network and $d_{M}$ is the maximum transmission range with maximum power
$P_{M}$ and zero interference.

$\triangle$ We prove a theoretical lower bound on the latencyenergy tradeoff under the physical interference model with SIC that, for any aggregation algorithm, the product of the energy consumption approximation ratio and the $(\alpha-1)^{\text {th }}$ power of the aggregation latency approximation ratio is lower bounded by $\Omega\left(\Delta^{\alpha-1}\right)$, where $\Delta$ is the maximum node degree (maximum number of nodes within the transmission range $d_{M}$ of any node).

$\triangle$ We propose EMA-SIC, an Energy-efficient Minimumlatency Aggregation algorithm under the physical interference model with SIC. As compared to existing work [3], [11] on minimum-latency data aggregation under the physical interference model, EMA-SIC can significantly lower the upper bound of the aggregation latency, to $O(D)$, and at the same time achieves an energy consumption approximation ratio that is the lowest possible with respect to the latency-energy tradeoff lower bound. In other words, our proposed algorithm achieves the asymptotical optimum in both aggregation latency and latency-energy tradeoff.

The remainder of the paper is organized as follows. We discuss related work in Sec. 2, and present the problem model in Sec. 3. We study the theoretical lower bounds for aggregation latency, energy consumption and their tradeoff in Sec. 4. The EMA-SIC algorithm and its analysis are presented in Sec. 5 and 6, respectively. The latency-energy efficiency of EMA-SIC is further studied via extensive simulations in Sec. 7. Finally, we conclude the paper in Sec. 8.

\section{Related Work}

\subsection{Minimum-Latency Data Aggregation}

There is a large body of literature on data aggregation in wireless sensor networks [1]-[3], [11], [16]-[19]. Most of the work target at minimum aggregation latency, without much consideration of the energy consumption. The current best upper bound on aggregation latency is $O(\Delta+R)$, which is based on the protocol interference model [1], [2], [16][18], where $R$ is the network radius in hops and $\Delta$ is the maximal node degree.

The paper [1] is the first work that achieves the $O(\Delta+R)$ aggregation latency upper bound. In [2], the minimumlatency data aggregation problem in a multihop wireless sensor network with the assumption that each node has a unit transmission range and an interference range of $\rho \geq 1$ is studied. $\mathrm{Xu}$ et al. [17] propose an aggregation schedule based on a distributed algorithm, which achieves a guaranteed maximum aggregation latency of $16 R+\Delta-14$; they also prove a lower bound of $\max \left\{R, \log _{2} n\right\}$ on the aggregation latency for any interference model, where $n$ is the network size. Different from the above work where connected dominating sets or maximal independent sets are employed, a novel approach of distributed aggregation with latency bound in $O\left(\Delta+R^{\prime}\right)$ is introduced in [16] by clustering. Here, $R^{\prime}$ is the inferior network radius satisfying that $R^{\prime} \leq R \leq D \leq 2 R^{\prime}$ with $D$ as the network diameter in hop-count. The MLAS problem is extended to the case with multiple sinks in [19] with latency bound of $O(\Delta+k R)$, where $k$ is the number of sinks. 
To the best of our knowledge, only two papers, [3] and [11], assume the physical interference model. A distributed aggregation scheduling algorithm with constant power assignment is proposed in [3], which achieves a latency upper bound of $O(\Delta+R)$. Li et al. [11] present a distributed algorithm with a latency bound of $O(K)$, where $K$ is the logarithm of the ratio between the length of the longest link and that of the shortest link, and a centralized solution with an aggregation latency of $O\left(\log ^{3} n\right)$ which is the current best result among all proposed aggregation algorithms under the physical interference model. However, no limit on the power is assumed in [11].

\subsection{Latency-Energy Tradeoffs in Data Aggregation}

The existence of a tradeoff between energy consumption and aggregation latency in wireless sensor networks is widely recognized. There were some attempts targeting at efficient data aggregation algorithms with both low aggregation latency and low energy usage [4]-[7], [20], but all are based on the protocol interference model expect [20] which only considers primary interference without mutual interference from other concurrent transmissions.

Yu et al. [4] explore the latency-energy tradeoff using techniques such as modulation scaling; algorithms are proposed to minimize the total energy consumption subject to a specified latency constraint. Arumugam et al. [5] propose a TDMA-based algorithm to effectively aggregate data in an energy-efficient way. In [6], the source node can specify its interest in minimizing energy consumption and/or source-to-sink delay, as input to the aggregation algorithm. The theoretical analysis in [7] demonstrates that there exists a latency-energy tradeoff in sensor data aggregation; an aggregation algorithm is designed, which achieves the asymptotical optimum for the tradeoff under the protocol interference model. To address the latency-energy tradeoff for in-network computation, of which data aggregation is a special case, an algorithm with order-optimal energy usage under given latency constraint is proposed in [20]. However, its order-optimality is derived over a network of uniformly random node distribution with simplified interference model as discussed previously, while not guaranteed over arbitrary network topologies.

To the best of our knowledge, there has been no work addressing the tradeoff under the physical interference model, not to mention the case with arbitrary network topologies and/or SIC technique, which is targeted in this paper.

\subsection{Successive Interference Cancellation}

The techniques of successive interference cancellation have been exploited in recent years. Weber et al. [15] have analyzed the transmission capacity of wireless ad-hoc networks using SIC, with both upper bound and lower bound in closed form. Simeone et al. [21] analyze the capacity of linear two-hop mesh networks with SIC; a decode-and-forward relaying mechanism is proposed by exploiting the possible relevant inter-cell channel gains and rate splitting with SIC.

Wang et al. [22] present a polynomial-time heuristic algorithm to approximate the optimal network throughput in ad hoc networks with joint routing and scheduling using SIC.
Lv et al. [23] propose simultaneity graph to characterize the effect of SIC on link dependence due to interference, and present an independent set based greedy scheme to construct a maximal feasible schedule. Jiang et al. [24] advocate the use of joint SIC and interference avoidance and introduce a cross-layer optimization framework for the joint scheme. In [25], a SIC-based scheduling algorithm, with polynomialtime complexity, is proposed to find short schedules for networks with arbitrary distribution in the Euclidean plane. However, none of the above considers the decoding delay with SIC. Our paper addresses this issue.

\section{The Problem Model}

We consider a multi-hop wireless sensor network with $n$ arbitrarily distributed sensor nodes $v_{0}, v_{1}, \ldots, v_{n-1}$ and a sink node $v_{n}$. The directed graph $G=(V, E)$ denotes the tree constructed for data aggregation from the sensor nodes to the sink, where $V=\left\{v_{0}, v_{1}, \ldots, v_{n}\right\}$ is the set of all nodes, and $E=\left\{e_{0}, e_{1}, \ldots, e_{n-1}\right\}$ is the set of transmission links in the tree with $e_{i}$ representing the link from sensor node $v_{i}$ to its parent. Without loss of generality, we assume that the minimum Euclidean distance between each pair of nodes is 1 .

We consider a time-slotted system. The transmission delay of one packet and the decoding delay to cancel one more signal with SIC are normalized to 1 time unit and $\tau$ time unit, respectively. The actual length of any time slot $t$ is $\left(1+\chi_{t} \cdot \tau\right)$ time units, with $\chi_{t}$ as the maximum number of canceled signals at any scheduled receiver in this slot depending on whether the SIC technique is applied: $\chi_{t}=0$ if there is no SIC application, while $\chi_{t}>0$ otherwise.

\subsection{The Data Aggregation Problem}

The data aggregation problem is to use the links in $E$ to construct a suitable tree and to design a correct and collisionfree aggregation schedule $\mathcal{S}=\left\{S_{0}, S_{1}, \ldots, S_{T-1}\right\}$, where $T$ is the total time slots for the schedule and $S_{t}$ denotes the subset of links in $E$ scheduled to transmit in time slot $t, t=0, \ldots, T-1$. A correct aggregation schedule must satisfy the following conditions. First, any link should be scheduled exactly once, i.e., $\bigcup_{t=0}^{T-1} S_{t}=E$ and $S_{i} \cap S_{j}=\emptyset$ where $i \neq j$. Second, primary interference (that due to a node acting as a transmitter and a receiver in the same time slot) should be avoided; that is, $T\left(S_{t}\right) \cap R\left(S_{t}\right)=$ $\emptyset, \forall t=0, \ldots, T-1$, where $T\left(S_{t}\right)$ and $R\left(S_{t}\right)$ denote the transmitter set and receiver set for the links in $S_{t}$, respectively. Third, a non-leaf node $v_{i}$ transmits to its parent only after all the links in the subtree rooted at $v_{i}$ have been scheduled, i.e., $T\left(S_{i}\right) \cap R\left(S_{j}\right)=\emptyset$ where $i<j$; in this way, $v_{i}$ can conduct local process to aggregate all data from its subtree (e.g. local maximum temperature of its subtree) and transmits the aggregated data only once to save energy. An aggregation schedule is collision-free if each scheduled transmission in time slot $t$, i.e., $\forall e_{i} \in S_{t}$, can be correctly received by its receiver according to the interference model in Sec. 3.3, $\forall t=0, \ldots, T-1$. Our objective is to minimize the aggregation latency, i.e., the overall time units of all slots, as well as the latency-energy tradeoff. Note that the aggregation latency already includes 


\begin{tabular}{|l|l||l|l|}
\hline$\alpha$ & Path loss ratio, Sec. 1 & $D$ & Network diameter in terms of hop count, Sec. 1 \\
\hline$\beta$ & SINR threshold, Sec. 1 & $n_{m i s}$ & Size of maximum independent set, Sec. 1 \\
\hline$N_{0}$ & Ambient noise power, Sec. 1 & $X$ & Max. cancelable signals at one receiver, Sec. 1 \\
\hline$P_{M}$ & Max. transmission power, Sec. 1 & $\chi_{t}$ & Max. canceled signals at each receiver, slot $t$, Sec. 3 \\
\hline$d_{M}$ & Max. transmission range with $P_{M}$, Sec. 1 & $\tau$ & Decoding delay to cancel one more signal, Sec. 3 \\
\hline$\Delta$ & Max. node degree, Sec. 1 & $h$ & Side length of hexagons, Sec. 5 \\
\hline$n$ & Number of sensor nodes in network, Sec. 1 & $n_{c d s}$ & Size of connected dominating set, Appendix I \\
\hline
\end{tabular}

TABLE 1: Notation table.

the end-to-end transmission delay, decoding delay with SIC and the cumulative queueing delay, since it is defined as the time-span between the time-point of first transmission and that when the sink collects all data.

\subsection{Decoding delay with SIC}

With the SIC technique for ZigBee standard in [13], one packet typically has a length of 128 bytes, which are modulated into 4096 physical-layer symbols. Symbols of each signal are decoded and canceled sequentially, with the requisite that three consecutive symbols should be buffered for each canceled symbol. The decoding delay for each canceled signal is the time span for 3 symbols, which is $\tau=\frac{3}{4096}$ of the transmission delay. If we have $\chi_{t}$ signals to cancel out, the total decoding delay is $\frac{3 \chi_{t}}{4096}$ time units while the saved transmission delay is $\chi_{t}$ time units, i.e., one time unit for each canceled signal. Thus, SIC has great potential in reducing the aggregation latency in wireless sensor networks. The above setting for decoding delay is also applied in our simulation study in Sec. 7 .

\subsection{Interference and Energy Models}

We adopt the physical interference model with the application of successive interference cancellation. With SIC, a receiver can recover multiple signals from simultaneous transmitters from the mixed signal received, as long as an interference cancellation sequence of the signals can be determined. The sequence is such that the $i^{\text {th }}$ signal remains strong enough, as judged by condition (2), after the previous $i-1$ signals have been removed (canceled) from the mixed signal. If SIC is not applied at a receiver, the receiver can recover at most one signal (from one sender) in each time slot, subject to condition (1).

In our study, we use the energy model that the power attenuation along each transmission link of length $r$ is proportional to $r^{\alpha}, \alpha \geq 2$, i.e., the received power is $P / r^{\alpha}$ if the sender uses transmission power $P$.

Let $P_{i}$ denote the transmission power used by node $v_{i}, i=0, \ldots, n-1$, and the maximum transmission power at any sensor node be $P_{M} \cdot{ }^{1}$ We assume no isolated node, i.e. each node can transmit to at least one other node in the network if the power level of $P_{M}$ is used. Let $D$ be the network diameter, which is in hops instead of the geometric distance, and is defined as the maximum of the minimum number of hops between any pair of nodes when the nodes are transmitting using maximum power $P_{M}$; and $d_{M}$ be the maximum transmission range of a node when using $P_{M}$ with zero interference.

Important notations used in the paper are summarized in table 1 with descriptions and places of first appearances.

1. We consider homogeneous networks with identical maximum transmission power on each node.

\section{THEORETICAL LOWER BOUNDS}

We first investigate the theoretical lower bounds on the aggregation latency, the energy consumption and the latencyenergy tradeoff for the data aggregation problem, respectively.

\subsection{Energy Consumption Bound}

We prove in Theorem 1 a lower bound, applicable to both the case with and that without SIC, on the overall energy consumption for data aggregation under the physical interference model.

Definition 1. (Maximum Independent Set with $P_{M}$ ) An independent set with $P_{M}$ in a wireless sensor network $G$ is a subset of nodes in the network graph, such that no node in the set can successfully transmit to another node in the set using the maximum power $P_{M}$ with zero interference, and a maximum independent set with $P_{M}$ is the largest such independent set in the graph, i.e., it has the maximum number of nodes.

Theorem 1. (Energy Consumption Lower Bound) Suppose the size of the maximum independent set with $P_{M}$ containing the sink in a wireless sensor network is $n_{m i s}+1$. The overall energy consumption for data aggregation in the network under the physical interference model, with or without SIC, is lower-bounded by $N_{0} \beta \frac{\left(n_{m i s} d_{M}\right)^{\alpha}}{n^{\alpha-1}}$.

We prove Theorem 1 by analyzing the energy consumption when links are scheduled in a "TDMA" fashion, i.e., only one link is scheduled to transmit in each time slot, and the data aggregation tree is a minimum spanning tree of the network, of which the weight of link $e_{i}$ is $d_{i i}^{\alpha}$, where $d_{i i}$ is the Euclidean length of link $e_{i}$. More details can be found in Appendix A.

\subsection{Aggregation Latency Bound}

In their recent work, $\mathrm{Xu}$ et al. [17] give a latency lower bound of $\Omega(D+\Delta)$ on sensor data aggregation under the protocol interference model, and a latency lower bound of $\Omega\left(\max \left\{R, \log _{2} n\right\}\right)$ under any interference model. We next prove an aggregation latency lower bound under the physical interference model with SIC.

Theorem 2. (Aggregation Latency Lower Bound) The latency of data aggregation in a wireless sensor network under the physical interference model with SIC, is lower-bounded by $\Omega\left(\max \left\{D, \log _{X+1} n\right\}\right)$, where $X=\left\lfloor\log _{1+\beta} \frac{P_{M}}{N_{0} \beta}+1\right\rfloor$.

We first demonstrate that $X$ is the maximum cancelable signals at one receiver, and then prove Theorem 2 by showing that the aggregation latency lower bound is achieved using maximum transmission power with $D, n$ and $X$ as dominant factors. Detailed proof is in Appendix B. 


\subsection{Latency-Energy Tradeoff}

The lower bounds on aggregation latency and overall energy consumption, as just derived, may not be achievable concurrently: the lower bound on energy consumption given in Theorem 1 is achieved only when the aggregation tree is a minimum spanning tree of the network and exactly one transmission along the tree is scheduled in each time slot. In this case, the aggregation latency is $n$. On the other hand, to achieve the lower bound on aggregation latency of $\Omega\left(\max \left\{D, \log _{X+1} n\right\}\right)$ as given in Theorem 2, larger powers up to $P_{M}$ at the transmitters may need to be used. Consequently, the tradeoff between aggregation latency and energy consumption needs to be addressed in the design of any data aggregation algorithm, which is the main objective of this paper.

Theorem 3 presents a theoretical lower bound on the combined performance of aggregation latency and energy consumption. The theorem is not to establish a definition for the latency-energy tradeoff, which may vary with different application concerns. However, the result in Theorem 3 can serve as a metric to examine whether an algorithm has achieved the best it can do in terms of both aggregation latency and energy consumption, with a tradeoff in between. A similar metric can be found in [7] under the protocol interference model.

Theorem 3. (Latency-Energy Tradeoff Lower Bound) Let $\rho_{L}$ and $\rho_{E}$ denote the approximation ratios of the aggregation latency and energy consumption with regard to the lower bounds in Theorems 1 and 2, respectively, with any given data aggregation algorithm. The product of the energy consumption approximation ratio, i.e., $\rho_{E}$, and the $(\alpha-1)^{\text {th }}$ power of the aggregation latency approximation ratio, i.e., $\rho_{L}^{\alpha-1}$, is lowered-bounded by $\Omega\left(\Delta^{\alpha-1}\right)$ in wireless networks under the physical interference model with SIC.

To prove the theorem, we show that there exists a sample network of $n+1$ nodes with maximum node degree $\Delta$ such that $\rho_{L}^{\alpha-1} \rho_{E}$ is lowered-bounded by $\Omega\left(\Delta^{\alpha-1}\right)$ under the physical interference model with SIC. See more details in Appendix C.

We will show in Sec. 6 that, our algorithm, to be proposed in Sec. 5, is asymptotically optimal in both aggregation latency and latency-energy tradeoff, with respect to the lower bounds in Theorems 1, 2 and 3 .

\section{EMA-SIC: ENERGY-EFFICIENT MINIMUM- LATENCY DATA AGgREGATION ALGORITHM WITH SUCCESSIVE INTERFERENCE CANCEL- \\ LATION}

We now design our EMA-SIC algorithm, which can outperform any other existing algorithm by its reduced maximum aggregation latency and asymptotically optimal latencyenergy tradeoff. It uses the successive interference cancellation technique, and consists of two parts: tree construction (T) and link scheduling (S).

\subsection{Tree Construction with SIC}

The aggregation tree construction in EMA-SIC comprises three steps, executed in a distributed fashion.
T1) Breadth-first search is launched by the sink $v_{n}$ to find a spanning tree of the network rooted at it, based on maximum transmission ranges of the nodes. Each node is assigned a level, indicating its hop-count to the sink. The sink node is initialized with level 0 .

T2) A connected dominating set (CDS) of the network (see Definition 2) is identified in the breath-first spanning (BFS) tree, by treating the sink as the first dominator and then finding other dominators using the most widely adopted algorithm, which is distributed, in [26]. This algorithm is executed in two phases to find the connected dominating set. In the first phase, a maximum independent set is constructed such that the distance between any pair of its complementary subsets is exactly two hops. Based on the constructed MIS, the second phase generates a connected dominating set by strategically selecting nodes to be added to or removed from the MIS. A tree rooted at the sink and connecting all other nodes in the connected dominating set can be built, such that each node in level $l \geq 1$ connects to its parent node in level $l-1$ of the BFS tree.

Definition 2. (Connected Dominating Set with $P_{M}$ ) A dominating set with $P_{M}$ in a wireless sensor network $G$ is a subset of nodes in the graph, such that every node outside the set can successfully transmit to at least one node in the set using the maximum power $P_{M}$. The nodes in a dominating set are referred to as dominators, and those not in the set are dominatees. A connected dominating set with $P_{M}$ is a dominating set within which any node can transmit to at least another node using $P_{M}$.

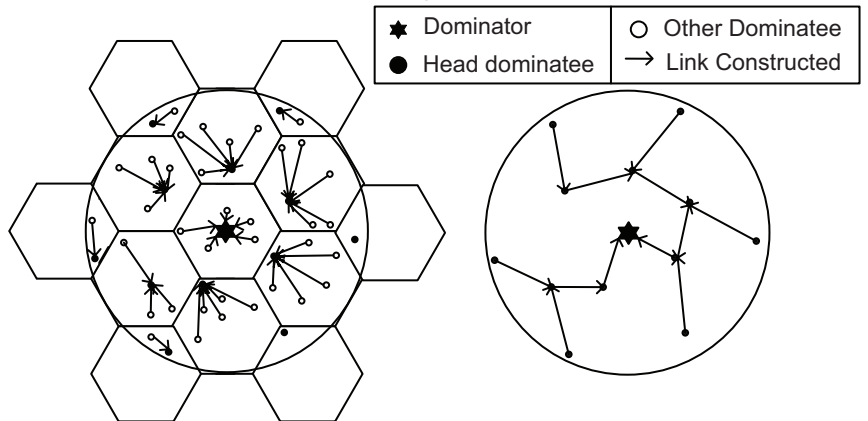

(a) Concurrent transmissions to the head dominatee.

(b) MST rooted at the dominator.

Fig. 2: The third step of tree construction in EMA-SIC: an example with one dominator.

T3) This step consists of two phases. Consider the dominators in the connected dominating set derived in the previous step. In the first phase, i.e., step T3.a, each dominator finds a disk centered at itself with radius equal to the maximum transmission range $d_{M}$, and use equalsized hexagons to cover the disk. An example is given in Fig. 2(a). The side length of the hexagons is $h=$ $\min \left\{d_{1}, d_{2}\right\}$ with $d_{1}=\frac{-1+\sqrt{1+4 / 3\left(1+\log _{1+\beta} \sqrt{P_{M} /\left(N_{0} \beta\right)}\right.}}{2}$ and $d_{2}=\frac{1}{2}\left(\frac{P_{M}}{N_{0} \beta}\right)^{\frac{1}{2 \alpha}}$, which are carefully assigned to ensure the validness of our algorithm, i.e., $K_{1}>0$ which is a parameter to be introduced shortly, proven in Appendix D. In each hexagon, the dominatee which is closest to the dominator is chosen as the head dominatee. We prove in Sec. 6 that, with our assignment of the hexagon side length, all dominatee nodes in a hexagon can concurrently transmit 
to the head dominatee which can successfully recover these transmissions using successive interference cancellation.

Note that, each dominator only needs the location information of the dominatees within the disk as in Fig. 2. Besides, the relative coordinate $(x, y)$ of each dominatee with its dominator as the origin $(0,0)$, is adequate to decide which hexagon it resides in and whether it is the head dominatee, without the use of absolute coordinates in the global view. So the step T3.a can be executed in a fully distributed fashion on each dominator just with relative locations of its dominatees.

In the next phase, i.e., step T3.b, for the much sparser topology consisting of only the head dominatees and the dominators, a local minimum spanning tree (MST) is built to connect the head dominatees to each dominator, as shown in Fig. 2(b). In constructing the MST, a link with length $r$ has weight $r^{\alpha}$, which reflects the power attenuation along the link. Using the connected dominating set as the backbone, a data aggregation tree of the entire network is formed.

In the above procedure, if a dominatee happens to reside in the overlapping area of hexagons or disks belonging to different dominators, it chooses to join the tree construction of the dominator geometrically closest to the sink.

Fig. 12 in Appendix E illustrates the tree construction procedure with an example.

\subsection{Link Scheduling with SIC}

The aggregation schedule consists of three steps: (S1) schedule the transmissions in individual hexagons from the non-head dominatees to the head dominatee; (S2) schedule the transmissions from head dominatees to their dominators along the local minimum spanning trees; (S3) schedule the aggregation transmissions of the dominators along the tree connecting them to the sink.

For step S1, within each hexagon, all the non-head dominatees transmit concurrently to the head dominatee. In order for the head dominatee to recover all the transmissions correctly, the transmission power for the $i^{\text {th }}$ link in the cancellation sequence, which has a link length of $d_{i i}$, is assigned to be $\left(N_{0}+I\right) \beta(1+\beta)^{X^{\prime}-i} d_{i i}^{\alpha}$, where $I=\frac{P_{M} /(2 h)^{\alpha}}{\beta(1+\beta)^{X^{\prime}-1}}-N_{0}$ is the upper bound of the cumulative interference from current transmissions in other hexagons and $X^{\prime}=3 h^{2}+3 h$ is the maximum number of non-head dominatees in any hexagon. The detailed derivation of $I$, $X^{\prime}$ and the power assignment can be found in the proof of Theorem 4 in Sec. 6 to show the correctness of EMA-SIC.

To alleviate inter-hexagon interferences, a schedule of hexagons is designed following the rule that the head dominatees in any two hexagons, of which the transmissions are scheduled in the same time slot, should be separated by a distance of greater than $K_{1}+1$ times the maximum link length $2 h$ in a hexagon, where $K_{1}=\left(6 X^{\prime}\right)^{\frac{1}{\alpha}}(1+$ $\left.\left(\frac{2}{\sqrt{3}}\right)^{\alpha} \frac{1}{\alpha-2}\right)^{\frac{1}{\alpha}}\left(\frac{1}{\beta(1+\beta)^{X^{\prime}-1}}-\frac{N_{0}(2 h)^{\alpha}}{P_{M}}\right)^{-\frac{1}{\alpha}}$.

To achieve collision-free link scheduling in steps S2 and S3, we apply the following two rules: (i) any two concurrent transmitters should be separated by a distance of at least $\left(K_{2}+1\right) d_{M}$, with $K_{2}=\left(6 \beta\left(1+\left(\frac{2}{\sqrt{3}}\right)^{\alpha} \frac{1}{\alpha-2}\right)+1\right)^{1 / \alpha}$, in order to bound the cumulative interference at each receiver; (ii) the transmission power for a link of length $r$ is set to $N_{0} \beta\left(2-1 / K_{2}^{\alpha}\right) r^{\alpha}$. This idea of separating concurrent transmitters by a predefined distance was also employed in [27] which however did not consider background noise in the interference model (we do here).

Note that, similar approaches of covering the network with hexagons are also utilized in aggregation algorithms of [3] and [11], but with fundamental differences from ours in following ways: (i) in [3], the network is covered with equalsized grids just for link scheduling across different grids without contribution to tree construction while our application of hexagons both constructs the aggregation tree and schedules link transmissions within the same hexagon; (ii) in [11], hexagons with differentiated sizes are iteratively used to construct the tree but with unlimited power assignment while our paper shows practical concern with maximum transmission power and applies hexagons with unique sizes for only part of the tree construction; moreover, absolute locations of all nodes in the global view are required in [11] while our paper just needs a weaker condition of relative position of each dominatee to its dominator.

The EMA-SIC algorithm is summarized in Algorithm 1.

\section{AnALYSIS OF EMA-SIC}

We next prove the correctness, as well as the latency and energy efficiencies of our algorithm.

\subsection{Correctness}

Theorem 4. (Correctness of EMA-SIC) EMA-SIC constructs a data aggregation tree and achieves a correct and collision-free aggregation schedule under the physical interference model.

Proof Sketch: It is easy to see an aggregation tree rooted at the sink is correctly constructed from the tree construction algorithm in EMA-SIC. We prove that EMA-SIC achieves a correct and collision-free aggregation schedule (see Sec. 3.A) by presenting upper bounds for cumulative interferences at each receiver in each step of the link scheduling, and showing that each received signal in step $\mathrm{S} 1$ and in steps $\mathrm{S} 2$ and $\mathrm{S} 3$ of the link scheduling satisfies the SINR constraint in Eqn. (2) and (1) even with its corresponding upper bound of cumulative interferences, respectively. The detailed proofs can be found in Appendices $\mathrm{F}$ and $\mathrm{G}$, respectively.

\subsection{Energy and Latency Efficiencies}

We show that EMA-SIC outperforms any other algorithm as it can reduce the maximum aggregation latency to $O(D)$, while maintaining an energy consumption approximation ratio that is the lowest possible- $O\left(\Delta^{\alpha-1}\right)$.

Theorem 5. (Latency Efficiency with EMA-SIC) The aggregation latency with EMA-SIC in any given network with network diameter $D$, is upper-bounded by $O(D)$, and the aggregation latency approximation ratio (with respect to the lower bound in Theorem 2) is upper-bounded by $O(1)$.

We prove Theorem 5 by showing that the scheduling latencies for step S1 and S2 are bounded with constant values while step $\mathrm{S} 3$ has an upper-bounded latency in the order of $O(D)$. See detailed proof in Appendix $\mathrm{H}$. 


Algorithm 1 EMA-SIC Algorithm
Input: Node set $V$ and the sink node $v_{n}$.

Output: Aggregation tree $E$ and link schedule $S$.

1: Initialization: $E, S \leftarrow \emptyset$.

2: Step T1: Construct a BFS tree on $V$ rooted at $v_{n}$.

3: Step T2: Construct a CDS on the BFS tree which includes $v_{n}$; build a spanning tree of the dominators rooted at $v_{n}$; add tree links to $E$.

4: Step T3.a: Cover the network with hexagons and connect each non-head dominatee to its head dominatee in the hexagon; add links to $E$.

5: Step T3.b: For each dominator, construct a local MST of its head dominatees rooted at it; add tree links to $E$.

6: $d_{1}:=\frac{-1+\sqrt{1+4 / 3\left(1+\log _{1+\beta} \sqrt{P_{M} /\left(N_{0} \beta\right)}\right)}}{2} ; d_{2}:=$ $\frac{1}{2}\left(\frac{P_{M}}{N_{0} \beta}\right)^{\frac{1}{2 \alpha}} ; h:=\min \left\{d_{1}, d_{2}\right\} ; X^{\prime}:=3 h^{2}+3 h$; $I:=\frac{P_{M} /(2 h)^{\alpha}}{\beta(1+\beta)^{X^{\prime}-1}}-N_{0}$;

$K_{1}:=\left(6 X^{\prime}\right)^{\frac{1}{\alpha}}\left(1+\left(\frac{2}{\sqrt{3}}\right)^{\alpha} \frac{1}{\alpha-2}\right)^{\frac{1}{\alpha}}\left(\frac{1}{\beta(1+\beta)^{X^{\prime}-1}}-\right.$ $\left.\frac{N_{0}(2 h)^{\alpha}}{P_{M}}\right)^{-\frac{1}{\alpha}}$.

7: Step S1: Schedule transmissions in hexagons from nonhead dominatees to their head dominatees, such that any two concurrent receivers are separated by at least $2\left(K_{1}+1\right) h$, and the transmission power is $\left(N_{0}+I\right) \beta(1+$ $\beta)^{X^{\prime}-i} d_{i i}^{\alpha}$ for the $i^{\text {th }}$ link in the receiver's cancellation sequence with length $d_{i i}$; add schedule to $S$.

8: $K_{2}:=\left(6 \beta\left(1+\left(\frac{2}{\sqrt{3}}\right)^{\alpha} \frac{1}{\alpha-2}\right)+1\right)^{1 / \alpha}$.

9: Steps S2 \& S3: Schedule the link transmissions in the aggregation tree containing only head dominatees and dominators, such that any two concurrent transmitters are separated by at least $\left(K_{2}+1\right) d_{M}$ and transmission power is $N_{0} \beta\left(2-1 / K_{2}^{\alpha}\right) r^{\alpha}$ for a link of length $r$; add the schedule to $S$.

10: return $E$ and $S$

Theorem 6. (Energy Efficiency with EMA-SIC) The energy consumption approximation ratio, that is, the upper bound of the overall energy consumption with using EMASIC to the lower bound in Theorem 1, is upper-bounded by $O\left(\Delta^{\alpha-1}\right)$, in any given network with a maximum node degree of $\Delta$.

To prove Theorem 6, the upper bounds of energy consumption for step S1, S2 and S3 are analyzed and characterized with $n_{m i s}$, respectively. Detailed proof can be found in Appendix I.

The following corollary shows that the energy consumption approximation ratio in Theorem 6 is indeed tight, for any algorithm achieving the aggregation latency upper bound in Theorem 5 .

Corollary 1. (Asymptotic optimum with EMA-SIC) The aggregation latency and the latency-energy tradeoff with EMA-SIC in any given network, are asymptotically optimal, or equivalently, with $O(1)$ approximation ratios with respect to the lower bounds in Theorem 1,2 and 3 .

This corollary can be easily proved by checking the approximation ratios of aggregation latency and energy consumption of EMA-SIC in Theorem 5 and 6, as well as the latency-energy tradeoff lower bound in Theorem 3.

Comparing our analytical results in Theorems 5 and 6 with those in [11] and [3], we can see that EMA-SIC reduces the upper bound of the maximum aggregation latency to $O(D)$ (which is the current best result in literature), and at the same time achieves an approximation ratio of energy consumption that is the lowest possible (Corollary 1).

\section{Simulation RESULTS}

We have presented the asymptotic performance of EMASIC in terms of aggregation latency and energy consumption together with their tradeoff by analyzing the respective upper bounds and approximation ratios in the worst cases. In this section, we further investigate the latency-energy efficiencies of EMA-SIC in average cases by comparison with two distributed aggregation algorithms under the physical interference model: Li et al.'s algorithm in [3] and the CellAS algorithm in [11].

We conduct our simulation in the Sinalgo [28] simulation framework, a packet-level wireless network simulator for testing and validating network algorithms.

Using the similar setting as that in [11], we consider wireless sensor networks having 100 to 1000 nodes that are randomly distributed with Uniform, Poisson or Cluster distributions $^{2}$ in a square field with side length from 100 to 200 meters. ${ }^{3}$ Fig. 14 in Appendix J gives an illustration of network topologies with 100 nodes under different distributions. The power of the background noise $N_{0}$ is set to a constant $10^{-6}$ joule/timeunit. Since the path loss ratio $\alpha$ has a typical value between 2 and 6 and the SINR threshold $\beta$ is generally assumed to be larger than 1 [3], [11], $\alpha$ is assigned 3,4 and 5 in the various settings, while $\beta$ is set to $2,4,6$ and 10,15, 20 for the low SINR and high SINR scenarios, respectively. The maximum transmission powers for $\mathrm{Li}$ et al.'s algorithm and the EMA-SIC algorithm are assigned values that would result in a transmission range of 40 meters and can maintain the network connectivity with high probability. ${ }^{4}$ The maximum transmission power in Cell-AS algorithm is infinite since no power limitation is assumed [11]. The decoding delay with SIC is calculated as in Sec. 3.2. Each datum is an average of 100 trials.

Li et al.'s algorithm is only effective with uniform node distribution, network side length of 180 and 200, and $(\alpha, \beta)$ being the pairs $(4,2),(5,2),(5,4)$, and $(5,6)$, consistent with the report in [11].

We therefore compare the latency-energy performance of the three algorithms under those settings in Fig. 3-10. Complete simulation results with other node distributions, network side lengths and $(\alpha, \beta)$ value pairs can be found our technical report [29], due to space constraint.

\subsection{Aggregation latency and energy consumption}

Fig. 3 and Fig. $4^{5}$ show that EMA-SIC outperforms both the Cell-AS and $\mathrm{Li}$ et al.'s algorithms in aggregation latency in

2. Please refer to [11] for detailed explanation of each distribution.

3 . With given number of nodes in the network, varying the network scale will change the node density.

4. Disconnected networks are meaningless in our problem since the sink cannot receive the data from all sensor nodes.

5. Results with network side length 180 under similar settings of Fig. 37 can be found in Appendix K. 
all cases, while consuming similar levels of energy as $\mathrm{Li}$ et al.'s algorithm (their curves largely overlap in Fig. 4; they will be compared separately in Fig. 5), which are far lower than those of the Cell-AS algorithm. The high energy consumption of Cell-AS algorithm results from its assumption of unlimited transmission power in [11].

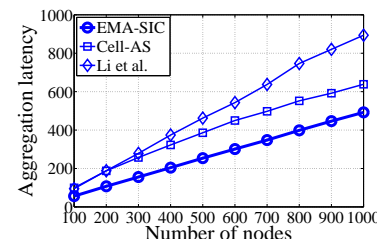

(a) $\alpha=4, \beta=2$

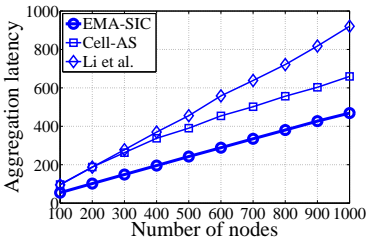

(c) $\alpha=5, \beta=4$

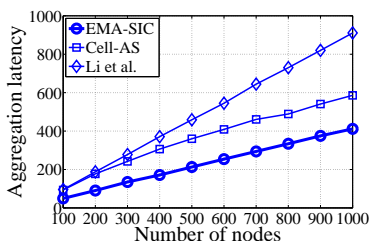

(b) $\alpha=5, \beta=2$

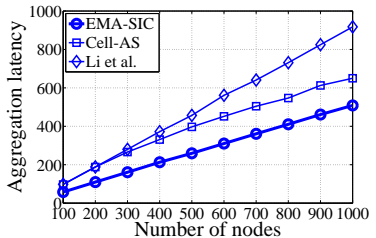

(d) $\alpha=5, \beta=6$
Fig. 3: Aggregation latency (time units) comparison under selected network settings in a $200 \times 200 \mathrm{~m}^{2}$ area.

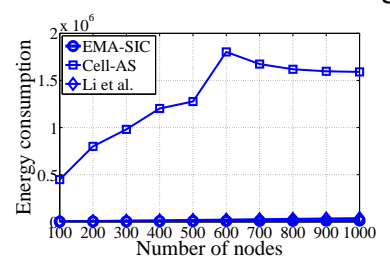

(a) $\alpha=4, \beta=2$

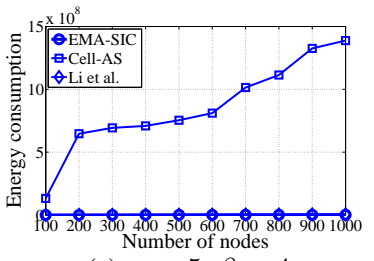

(c) $\alpha=5, \beta=4$

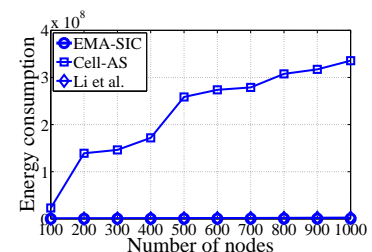

(b) $\alpha=5, \beta=2$

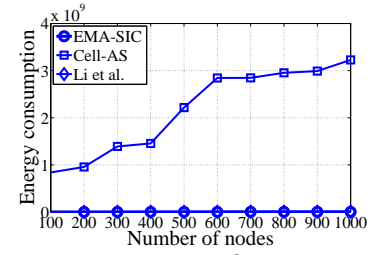

(d) $\alpha=5, \beta=6$
Fig. 4: Energy consumption (joule) comparison under selected network settings in a $200 \times 200 \mathrm{~m}^{2}$ area.

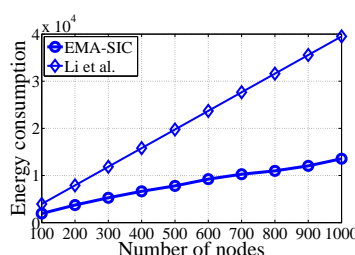

(a) $\alpha=4, \beta=2$

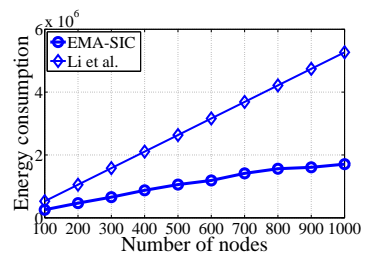

(c) $\alpha=5, \beta=4$

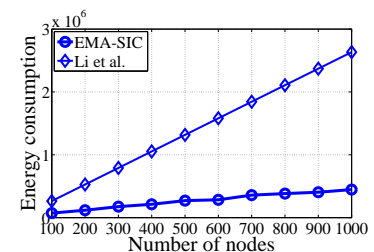

(b) $\alpha=5, \beta=2$

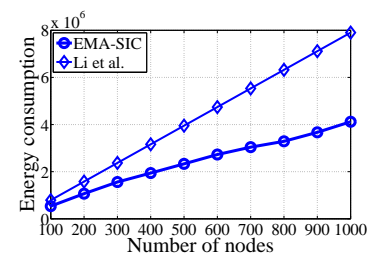

(d) $\alpha=5, \beta=6$
Fig. 5: A separate comparison of energy consumption (joule) between EMA-SIC and $\mathrm{Li}$ et al.'s algorithm under

selected network settings in a $200 \times 200 \mathrm{~m}^{2}$ area.

Fig. 4 also shows that the energy consumption of CellAS may go down when the number of nodes reaches 7001000 , which can be explained by the decrease of link lengths between nodes (thus decreased transmission power per link) when the node density in the same square area increases.

As stated previously, it is hard to tell the differences between the energy consumption curves of EMA-SIC and Li et al.'s algorithm in Fig. 4. Thus, we conduct a separate comparison of energy usage just between EMA-SIC and $L i$ et al.'s algorithm, and show that EMA-SIC is superior to Li et al.'s algorithm in energy consumption in Fig. 5. The curves of Li et al.'s algorithm are straight lines in Fig. 5 as a result of its constant power assignment in [3].

Another observation with Fig. 3-5 is that: (1) the aggregation latency of each algorithm is lower in settings of larger $\alpha$ (which means more path loss of power, and thus lower interference from other nodes) and smaller $\beta$ (corresponding to lower SINR requirement); (2) the energy consumption of each algorithm increases with the enhanced value of $\alpha$ (requiring higher transmission power to counteract the increased power loss and meet the SINR requirement) and $\beta$ (higher SINR requirement).

\subsection{Latency-energy tradeoff}

Next, we adopt the metric for latency-energy tradeoff as "the product of energy consumption and the $(\alpha-1)^{\text {th }}$ power of aggregation latency" to examine the performance of these algorithms in Fig. 6 for selected settings. The metric proposed in Theorem 3 equals to this revised metric divided by "the product of the optimal energy consumption and the $(\alpha-1)^{\text {th }}$ power of the optimal aggregation latency". Since the optimums of both aggregation latency and energy consumption of a given network should be the same for any aggregation algorithm, while the optimal aggregation latency of any given network is hard to find under the physical interference model (NP-hard), we adopt the revised metric here, which is equivalent to the previous metric multiplied by a constant factor.

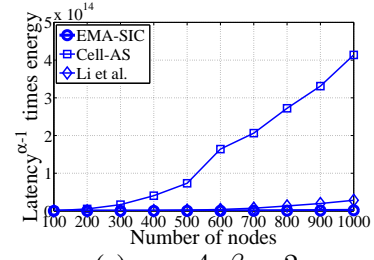

(a) $\alpha=4, \beta=2$

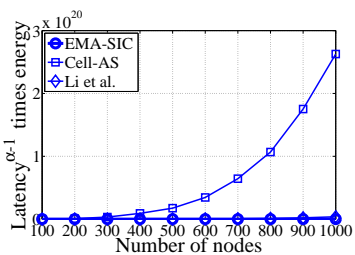

(c) $\alpha=5, \beta=4$

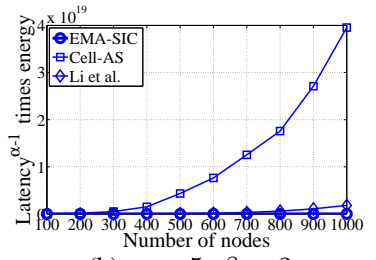

(b) $\alpha=5, \beta=2$

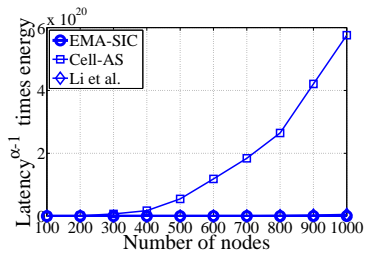

(d) $\alpha=5, \beta=6$
Fig. 6: Latency-energy tradeoff comparison under selected network settings in a $200 \times 200 \mathrm{~m}^{2}$ area.

It can be observed that, the Cell-AS algorithm has a significantly poorer latency-power tradeoff, compared with that of the other two algorithms. As the performance differences for Li et al.'s algorithm and the EMA-SIC algorithm are not distinguishable in Fig. 6, we present a separate comparison of latency-energy tradeoff between these two algorithms in Fig. 7. We can have that EMA-SIC algorithm achieves an evidently better latency-energy tradeoff, further confirming its superiority to other aggregation algorithms. 

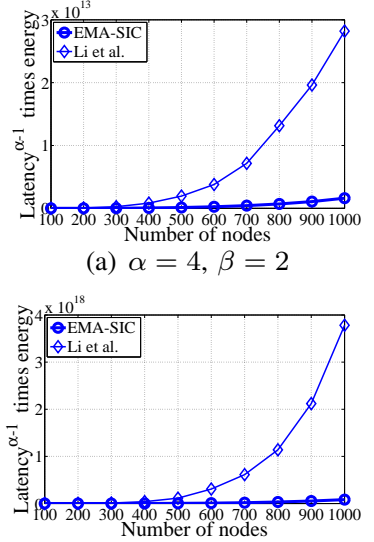

(c) $\alpha=5, \beta=4$ (a) $\alpha=4, \beta=2$

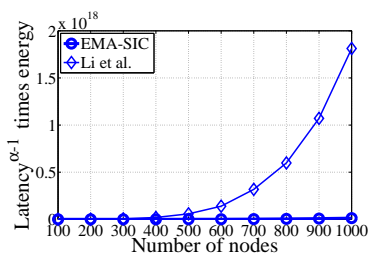

(b) $\alpha=5, \beta=2$

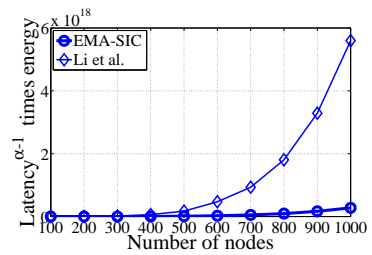

(d) $\alpha=5, \beta=6$
Fig. 7: A separate comparison of latency-energy between

EMA-SIC and $\mathrm{Li}$ et al.'s algorithm under selected network settings in a $200 \times 200 \mathrm{~m}^{2}$ area.

\subsection{Impact of network scale}

We also examine the impact of network scale on the latency-energy efficiency of the three algorithms in various network settings. Note that, since the network topologies are generated randomly, it is not straightforward to adjust the network diameter or node degree for performance comparison. Thus, we indirectly change the network diameter and node degree by varying the network scale. With the same number of nodes in the network, increasing the network scale will result in enhanced average network diameter while decreased average node degree.

We present results under selective settings, where $\mathrm{Li}$ et al.'s algorithm exerts its effectiveness, in Fig. 8-10 with uniformly distributed 1000 nodes. Detailed comparisons under other settings are included in technical report [29].

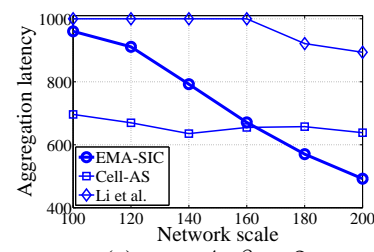

(a) $\alpha=4, \beta=2$

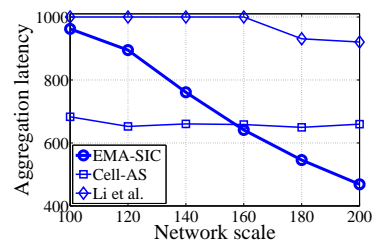

(c) $\alpha=5, \beta=4$

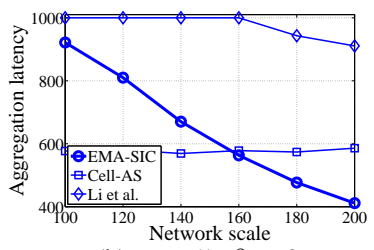

(b) $\alpha=5, \beta=2$

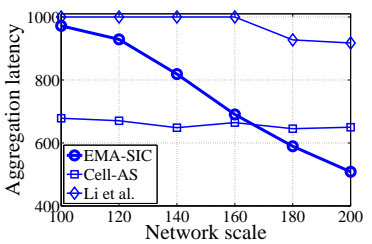

(d) $\alpha=5, \beta=6$
Fig. 8: Impact of network scale on the aggregation latency (time units) under selected network settings with uniform distribution and 1000 nodes.

With Fig. 8, we see that: i) Li et al.'s algorithm becomes effective when network scale reaches $180 \times 180$ square meters (detailed explanation in [11]); ii) Cell-AS algorithm has a relatively stable latency performance when network scale varies, which is due to its assumption of unlimited transmission power such that the network diameter and maximum node degree are fixed as 1 and $n$, respectively; and iii) the latency with EMA-SIC keeps decreasing when the size of network scales up (larger network diameter and smaller node degree), which can be understood that

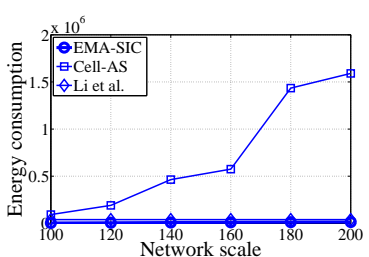

(a) $\alpha=4, \beta=2,3$ algorithms

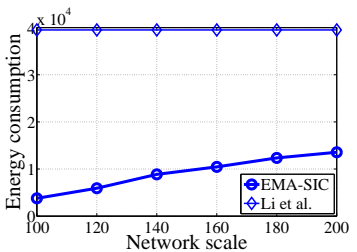

(c) $\alpha=4, \beta=2,2$ algorithms

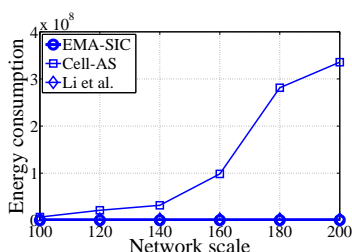

(b) $\alpha=5, \beta=2,3$ algorithms

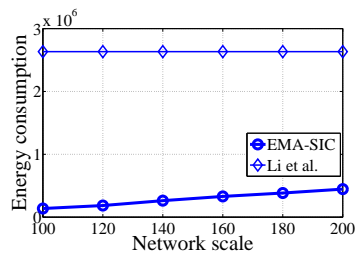

(d) $\alpha=5, \beta=2,2$ algorithms
Fig. 9: Impact of network scale on the energy consumption (joule) under selected network settings with uniform distribution and 1000 nodes.

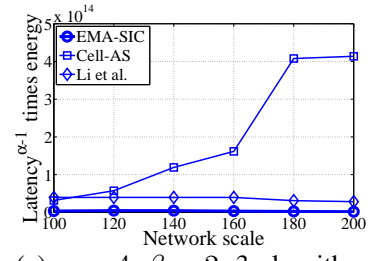

(a) $\alpha=4, \beta=2,3$ algorithms

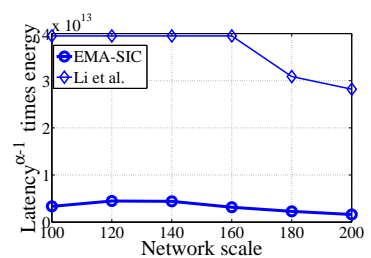

(c) $\alpha=4, \beta=2,2$ algorithms

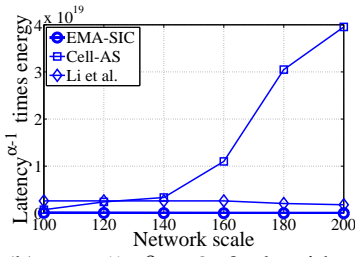

(b) $\alpha=5, \beta=2,3$ algorithms

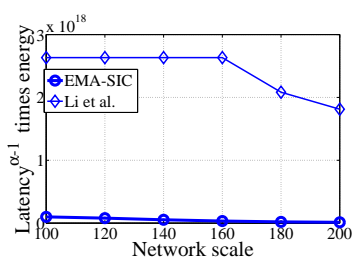

(d) $\alpha=5, \beta=2,2$ algorithms
Fig. 10: Impact of network scale on the latency-energy tradeoff under selected network settings with uniform distribution and 1000 nodes.

larger network scale leads to smaller node density and thus lower mutual interference among node pairs while more collision-free scheduling opportunities. Network diameter is the dominant factor for worst-case study to find theoretical bounds for aggregation latency as in Sec. 4 and 6. But, the impact of node degree overwhelms the network diameter on the average latency performance in random cases.

Although Cell-AS performs better, in aggregation latency, than EMA-SIC does when the network scale is smaller than $160 \times 160$ square meters, we argue that the impractical assumption of unlimited power by Cell-AS algorithm will render it unapplicable in power-constraint cases (wireless sensor networks typically belong to these cases). In contrast, EMA-SIC fits well in all network settings, with any given maximum transmission power. Besides, EMA-SIC strictly outperforms Cell-AS in energy consumption and latencyenergy tradeoff as shown in Fig. 9 and 10.

In Fig. 9 and 10, we present the simulation results for $(\alpha, \beta) \in\{(4,2),(5,2)\}$ while that for $(\alpha, \beta) \in$ $\{(5,4),(5,6)\}$ have similar conclusions and are included in Appendix $\mathrm{K}$ due to space constraint. With Fig. 9.a and 9.b, we have that the Cell-AS algorithm consumes much higher energy, proportional to the network scale, than the others, as a consequence of its unlimited power assumption. In Fig. 9.c and 9.d, we conduct a separate comparison 
on energy consumption between EMA-SIC and $\mathrm{Li}$ et al.'s algorithm. We can see that: i) Li et al.'s algorithm has the same energy consumption in various network scales as a result of its constant power assignment; and ii) EMASIC consumes significantly lower energy, also proportional to the network scale (which is a natural result because of the increased distances among node pairs in networks with larger scale). Fig. 10.a and 10.b present the latency-energy tradeoff by the three algorithms. Cell-AS algorithm has the poorest tradeoff performance, which scales up with the network side length, while Fig. 10.c and 10.d demonstrate that EMA-SIC algorithm also remarkably outperforms $\mathrm{Li}$ et al.'s algorithm in latency-energy tradeoff.

\section{Concluding Remarks}

This paper investigates the latency-energy tradeoff of data aggregation in wireless sensor networks under the physical interference model and using the successive interference cancellation (SIC) technique. We derive the theoretical lower bounds on both aggregation latency and energy consumption as well as their tradeoff, and give an energy-efficient minimum-latency data aggregation algorithm (EMA-SIC) which can achieve the asymptotically optimal aggregation latency and latency-energy tradeoff. We show that the EMA-SIC algorithm has a constant approximation ratio of aggregation latency (with respect to the theoretical lower bound) while consuming the lowest possible amount of energy. We conduct simulation studies to further validate the superiority of EMA-SIC in terms of latency-energy performance over other work under the physical interference model. As our ongoing work, we plan to evaluate the latency-energy tradeoff in wireless sensor networks with dynamics, e.g. stochastic node sleep and wake up events, and heterogenous node capabilities, e.g. differentiated battery power.

\section{RefERENCES}

[1] S. C. H. Huang, P. Wan, C. T. Vu, Y. Li, and F. Yao, "Nearly constant approximation for data aggregation scheduling in wireless sensor networks," in Proc. of IEEE INFOCOM'07, 2007.

[2] P. J. Wan, S. C. H. Huang, L. X. Wang, Z. Y. Wan, and X. H. Jia, "Minimum-latency aggregation scheduling in multihop wireless networks," in Proc. of ACM MOBIHOC'09, 2009.

[3] X. Y. Li, X. H. Xu, S. G. Wang, S. J. Tang, G. J. Dai, J. Z. Zhao, and Y. Qi, "Efficient data aggregation in multi-hop wireless sensor networks under physical interference model," in Proc. of IEEE MASS'09, 2009.

[4] Y. Yu, B. Krishnamachari, and V. K. Prasanna, "Energy-latency tradeoffs for data gathering in wireless sensor networks," in Proc. of IEEE INFOCOM'04, 2004.

[5] M. Arumugam and S. S. Kulkarni, "Tradeoff between energy and latency for convergecast," in Proc. of IEEE INSS'05, 2005.

[6] H. M. Ammari and S. K. Das, "Trade-off between energy savings and source-to-sink delay in data dissemination for wireless sensor networks," in Proc. of ACM MSWiM'05, 2005.

[7] X. Y. Li, Y. Wang, and Y. Wang, "Complexity of data collection, aggregation, and selection for wireless sensor networks," IEEE Transactions on Computers, vol. 60, pp. 386 - 399, 2011.

[8] G. Brar, D. Blough, and P. Santi, "Computationally efficient scheduling with the physical interference model for throughput improvement in wireless mesh networks," in Proc. of ACM MOBICOM'06, 2006.

[9] D. Chafekar, V. S. Kumar, M. V. Marathe, S. Parthasarathy, and A. Srinivasan, "Approximation algorithms for computing capacity of wireless networks with sinr constraints," in Proc. of IEEE INFOCOM'08, 2008.
[10] T. Moscibroda, "The worst-case capacity of wireless sensor networks," in Proc. of ACM/IEEE IPSN'07, 2007.

[11] H. Li, Q.-S. Hua, C. Wu, and F. C. M. Lau, "Minimum-latency aggregation scheduling in wireless sensor networks under physical interference model," in Proc. of ACM MSWiM'10, 2010.

[12] J. Andrews, "Interference cancellation for cellular systems: A contemporary overview," IEEE Wireless Communications, vol. 12, pp. $19-29,2005$.

[13] D. Halperin, T. E. Anderson, and D. Wetherall, "Taking the sting out of carrier sense: Interference cancellation for wireless lans," in Proc. of ACM MOBICOM'08, 2008.

[14] IEEE Std. 802.15.4-2006: Wireless medium access control and physical layer specifications for low-rate wireless personal area networks, IEEE Std., 2003. [Online]. Available: http://www.ieee802.org

[15] S. P. Weber, J. G. Andrews, X. Y. Yang, and G. de Veciana, "Transmission capacity of wireless ad hoc networks with successive interference cancellation," IEEE Transactions on Information Theory, vol. 53, pp. $2799-2814,2007$.

[16] Y. Li, L. Guo, and S. K. Prasad, "An energy-efficient distributed algorithm for minimum-latency aggregation scheduling in wireless sensor networks," in Proc. of IEEE ICDCS'10, 2010.

[17] X. Xu, X.-Y. Li, X. Mao, S. Tang, and S. Wang, "A delay-efficient algorithm for data aggregation in multihop wireless sensor networks," IEEE Transactions on Parallel and Distributed Systems, vol. 22, pp. $163-175,2011$

[18] B. Yu, J. Li, and Y. Li, "Distributed data aggregation scheduling in wireless sensor networks," in Proc. of IEEE INFOCOM'09, 2009.

[19] B. Yu and J. Li, "Minimum-time aggregation scheduling in multi-sink sensor networks," in Proc. of IEEE SECON'11, 2011.

[20] P. Balister, B. Bollobas, A. Anandkumar, and A. Willsky, "Energylatency tradeoff for in-network function computation in random networks," in Proc. of IEEE INFOCOM'11, 2011.

[21] O. Simeone, O. Somekh, Y. Bar-Ness, H. V. Poor, and S. Shamai, "Capacity of linear two-hop mesh networks with rate splitting decodeand-forward relaying and cooperation," in Proc. of IEEE Allerton'07, 2007.

[22] X. Wang and J. Garcia-Luna-Aceves, "Embracing interference in ad hoc networks using joint routing and scheduling with multiple packet reception," in Proc. of IEEE INFOCOM'08, 2008.

[23] S. Lv, W. Zhuang, X. Wang, and X. Zhou, "Scheduling in wireless ad hoc networks with successive interference cancellation," in Proc. of IEEE INFOCOM'11, 2011.

[24] C. Jiang, Y. Shi, Y. T. Hou, W. Lou, S. Kompella, and S. F. Midkiff, "Squeezing the most out of interference: An optimization framework for joint interference exploitation and avoidance," in Proc. of IEEE INFOCOM'12, 2012

[25] O. Goussevskaia and R. Wattenhofer, "Scheduling wireless links with successive interference cancellation," in Proc. of IEEE ICCCN'12, 2012.

[26] P. J. Wan, K. M. Alzoubi, and O. Frieder, "Distributed construction of connected dominating set in wireless ad hoc networks," in Proc. of IEEE INFOCOM'02, 2002.

[27] L. Fu, S. C. Liew, and J. Huang, "Effective carrier sensing in csma networks under cumulative interference," in Proc. of IEEE INFOCOM'10, 2010.

[28] Sinaglo: Simulator for network algorithms. [Online]. Available: http://www.disco.ethz.ch/projects/sinalgo/

[29] H. Li, C. Wu, D. X. Yu, Q. S. Hua, and F. C. M. Lau, "Aggregation latency-energy tradeoff in wireless sensor networks with successive interference cancellation," Tech. Rep., http://i.cs.hku.hk/ hxli/SIC.pdf 2012.

[30] E. F. Beckenbach and R. Bellman, Inequalities. New York: SpringerVerlag, 1965

[31] H. Groemer, "Über die einlagerung von kreisen in einen konvexen bereich," Math. Z., vol. 73, pp. 285-294, 1960.

[32] C. Ambuhl, "An optimal bound for the mst algorithm to compute energy efficient broadcast trees in wireless networks," in Proc. of ICALP'05, 2005. 


\section{APPENDIX A}

\section{Proof to Theorem 1}

We prove the theorem based on a lemma.

Lemma 1. Consider the $n$ positive real numbers, $x_{1}, \ldots, x_{n}$, we have that,

$$
\sum_{i=1}^{n} x_{i}^{\alpha} \geq \frac{\left(\sum_{i=1}^{n} x_{i}\right)^{\alpha}}{n^{\alpha-1}}, \forall \alpha \geq 1
$$

Proof: Consider the generalized mean of $n$ positive real numbers, $x_{1}, \ldots, x_{n}$, with exponent $p$ (where $p$ is a non-zero real number): $M_{p}\left(x_{1}, \ldots, x_{n}\right)=\left(\frac{1}{n}\right.$. $\left.\sum_{i=1}^{n} x_{i}^{p}\right)^{1 / p}$. We use the generalized mean inequality [30], $M_{p}\left(x_{1}, \ldots, x_{n}\right) \leq M_{q}\left(x_{1}, \ldots, x_{n}\right)$ if $p<q$, where the two means are equal if and only if $x_{1}=x_{2}=\cdots=x_{n}$. Since $\alpha \geq 1$, we have

$$
\begin{aligned}
& M_{1}\left(x_{1}, \ldots, x_{n}\right) \leq M_{\alpha}\left(x_{1}, \ldots, x_{n}\right) \\
\Rightarrow & \frac{\sum_{i=1}^{n} x_{i}}{n} \leq\left(\frac{1}{n} \sum_{i=1}^{n} x_{i}^{\alpha}\right)^{1 / \alpha} \\
\Rightarrow & \sum_{i=1}^{n} x_{i}^{\alpha} \geq \frac{\left(\sum_{i=1}^{n} x_{i}\right)^{\alpha}}{n^{\alpha-1}} .
\end{aligned}
$$

Proof of Theorem 1: The minimum overall energy consumption for data aggregation is achieved when links are scheduled in a "TDMA" fashion, i.e., only one link is scheduled to transmit in each time slot (and there is no interference from concurrent transmissions) and the data aggregation tree is a minimum spanning tree of the network, of which the weight of link $e_{i}$ is $d_{i i}^{\alpha}$, where $d_{i i}$ is the Euclidean length of link $e_{i}$. Note that, even if the application of SIC is an option, the optimal choice for minimum overall energy consumption is not to use SIC while scheduling the links as above. The rationale is that, extra energy is required to recover the signal that is not the first canceled one, in order to compensate for the cumulative interference from those links canceled before it.

Thus, the transmission power $P_{i}$, which is also the energy consumption since the transmission delay is normalized to 1 time unit as in Sec. 3, to guarantee the success of transmission $e_{i}$ is such that

$$
\frac{P_{i} / d_{i i}^{\alpha}}{N_{0}} \geq \beta \Rightarrow P_{i} \geq N_{0} \beta d_{i i}^{\alpha} .
$$

The minimum distance between any two nodes in the maximum independent set with $P_{M}$ should be larger than $d_{M}$, the maximum transmission range between any two nodes. The overall length of edges in any tree connecting all the nodes in the maximum independent set with $P_{M}, L_{m i s}$, should be larger than $n_{m i s} d_{M}$. The overall link length in the minimum spanning tree in the network is no smaller than $L_{m i s}$, i.e., $\sum_{i=0}^{n-1} d_{i i} \geq L_{m i s}>n_{m i s} d_{M}$. By Lemma 1 , the overall energy consumption is lower-bounded by

$\sum_{i=0}^{n-1} P_{i} \geq N_{0} \beta \sum_{i=0}^{n-1} d_{i i}^{\alpha} \geq N_{0} \beta \frac{\left(\sum_{i=0}^{n-1} d_{i i}\right)^{\alpha}}{n^{\alpha-1}}>N_{0} \beta \frac{\left(n_{m i s} d_{M}\right)^{\alpha}}{n^{\alpha-1}}$.

\section{APPENDIX B}

\section{Proof to Theorem 2}

We prove the theorem based on Lemma 2 .
Lemma 2. Suppose a receiver can recover $X$ simultaneous signals in one time slot using SIC. The transmission power used at the transmitter of the $i^{\text {th }}$ signal in the interference cancellation sequence is at least $N_{0} \beta(1+\beta)^{X-i} d_{i i}^{\alpha}$, where $d_{i i}$ is the link length of the $i^{\text {th }}$ signal.

Proof: We prove this lemma by induction.

The base case: Consider the last signal to recover in the interference cancellation sequence, i.e., $i=X$. The signal is affected by background noise, but not any interference from concurrent transmissions. Its SINR value satisfies

$$
\frac{P_{X} / d_{X X}^{\alpha}}{N_{0}} \geq \beta \Rightarrow P_{X} \geq N_{0} \beta d_{X X}^{\alpha}=N_{0} \beta(1+\beta)^{X-X} d_{X X}^{\alpha} .
$$

Therefore, the lower bound holds for the base case.

Inductive step: Suppose the transmission power of the $j^{t h}$ signal, $P_{j}, j=i+1, \ldots, X$, is at least $N_{0} \beta(1+\beta)^{X-j} d_{j j}^{\alpha}$. Then the transmission power of the $i^{\text {th }}$ signal, $P_{i}$, satisfies

$$
\begin{aligned}
& \frac{P_{i} / d_{i i}^{\alpha}}{N_{0}+\sum_{j=i+1}^{X} P_{j} / d_{j j}^{\alpha}} \geq \beta \\
\Rightarrow & P_{i} \geq\left(N_{0}+\sum_{j=i+1}^{X} \frac{P_{j}}{d_{j j}^{\alpha}}\right) \beta d_{i i}^{\alpha} \\
\Rightarrow & P_{i} \geq\left(N_{0}+\sum_{j=i+1}^{X} N_{0} \beta(1+\beta)^{X-j}\right) \beta d_{i i}^{\alpha} \\
\Rightarrow & P_{i} \geq N_{0} \beta\left(1+\beta \frac{1-(1+\beta)^{X-i}}{1-(1+\beta)}\right) d_{i i}^{\alpha} \\
= & N_{0} \beta(1+\beta)^{X-i} d_{i i}^{\alpha} .
\end{aligned}
$$

The lemma is proved.

Proof of Theorem 2: In a multi-hop wireless sensor network with diameter $D$, the maximum number of hops for the data from a sensor node to reach the sink is $D$, when all intermediate nodes transmit using $P_{M}$. Therefore, at least $D$ time slots are needed. Let $T$ be the minimum number of time slots needed for data aggregation. We have $T \geq D$.

Suppose at most $X$ simultaneous transmissions can be successfully recovered at a receiver under the physical interference model with SIC, when the maximum transmission power of each sender is $P_{M}$. Consider the first signal in the interference cancellation sequence. Based on Lemma 2 and recall that the minimum distance between two nodes is 1 in our network model, we have

$$
P_{M} \geq N_{0} \beta(1+\beta)^{X-1} 1^{\alpha} \Rightarrow X=\left\lfloor\log _{1+\beta} \frac{P_{M}}{N_{0} \beta}+1\right\rfloor .
$$

Therefore, at most $X$ transmissions can be concurrently scheduled among every group of $X+1$ nodes. In a network with $n$ nodes, each of which needs to transmit exactly once, at most $n \frac{X}{X+1}$ nodes can be scheduled to transmit in the first time slot, while $\frac{n}{X+1}$ nodes remain. Therefore, in time slot $t(1 \leq t \leq T-1)$, at most $n\left(\frac{1}{X+1}\right)^{t-1} \frac{X}{X+1}$ nodes can be scheduled for transmission while $n\left(\frac{1}{X+1}\right)^{t}$ nodes remain. Finally at time slot $T$, only the sink node remains. We thus have $n\left(\frac{1}{X+1}\right)^{T} \leq 1$, which gives $T \geq \log _{X+1} n$.

Since the length of each time slot $t$ is $1+\chi_{t} \tau \geq 1$ time units, combining the two cases above, the aggregation latency is lower-bounded by $\Omega\left(\max \left\{D, \log _{X+1} n\right\}\right)$ time units. 


\section{APPENDIX C}

\section{Proof to Theorem 3}

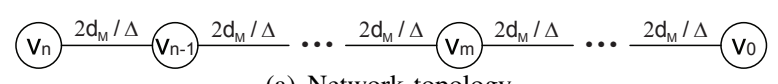

(a) Network topology.

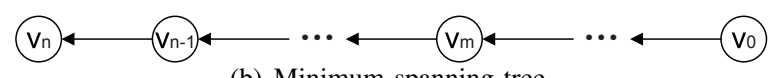

(b) Minimum spanning tree.

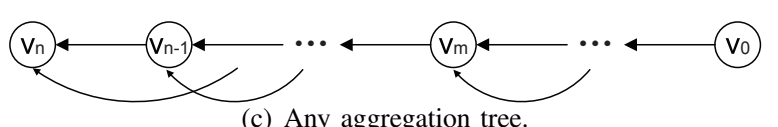

(c) Any aggregation tree.

Fig. 11: A chain network with $n+1$ nodes evenly distributed along a line.

Proof: To prove the theorem, we only need to show that there exists some network of $n+1$ nodes with maximum node degree $\Delta$ such that $\rho_{L}^{\alpha-1} \rho_{E}$ is lowered-bounded by $\Omega\left(\Delta^{\alpha-1}\right)$ under the physical interference model with SIC. We consider the chain network in Fig. 11(a), where $n+1$ nodes are evenly distributed along a line segment $\left[0, \frac{2 n d_{M}}{\Delta}\right]$, i.e., node $v_{i}$ is at position $\frac{2 d_{M}}{\Delta} \cdot(n-i), 0 \leq i \leq n$. So, the network diameter $D$ is $\frac{2 n}{\Delta}$ (the hop-count from $v_{0}$ to $v_{n}$ ), and the maximum node degree is exactly $\Delta, e . g$., the node degree of some intermediate node $v_{m}$. We further let $\Delta \leq 2 n / \log _{2} n$ in this network such that $\frac{2 n}{\Delta} \geq \log _{2} n \geq$ $\log _{X+1} n$, where $X=\left\lfloor\log _{1+\beta} \frac{P_{M}}{N_{0} \beta}+1\right\rfloor \geq 1$ as defined in Theorem 2. Consequently, the aggregation latency lower bound is $D=\frac{2 n}{\Delta}$ time units for this network. It is easy to see that the minimum energy data aggregation tree is simply the path $v_{0} v_{1} \ldots v_{n}$ as in Fig. 11(b), with the minimum energy consumption being $n N_{0} \beta\left(\frac{2 d_{M}}{\Delta}\right)^{\alpha}$.

For the general case where the data aggregation topology is a tree $T$ as in Fig. 11(c) with any given aggregation algorithm, we assume there are $k$ edges along the unique path from $v_{0}$ to $v_{n}$ in the tree. Then the data aggregation along tree $T$ takes at least $k$ time slots or equivalently $\sum_{t=1}^{k}\left(1+\chi_{t} \tau\right) \geq k$ time units. Denote the Euclidean lengths of the $k$ edges in $T$ by $r_{i}, i=1, \ldots, k$. Clearly, $\sum_{i=1}^{k} r_{i} \geq$ $\frac{2 n d_{M}}{\Delta}$. Then the energy consumption along this path is at least $\sum_{i=1}^{k} N_{0} \beta r_{i}^{\alpha} \geq N_{0} \beta \frac{\left(\sum_{i=1}^{k} r_{i}\right)^{\alpha}}{k^{\alpha-1}} \geq N_{0} \beta \frac{\left(2 n d_{M}\right)^{\alpha}}{k^{\alpha-1} \Delta^{\alpha}}$, where the first inequality is based on Lemma 1 . Then, for any given aggregation algorithm, we have that

$$
\begin{aligned}
\rho_{L}^{\alpha-1} \rho_{E} & \geq\left(\frac{k}{2 n / \Delta}\right)^{\alpha-1} \frac{N_{0} \beta\left(2 n d_{M}\right)^{\alpha} /\left(k^{\alpha-1} \Delta^{\alpha}\right)}{n N_{0} \beta\left(2 d_{M} / \Delta\right)^{\alpha}} \\
& =(\Delta / 2)^{\alpha-1} .
\end{aligned}
$$

\section{APPENDIX D \\ THE VALIDITY OF HEXAGON SIDE LENGTH}

With Eqn. (3) in Appendix B, we can have that $K_{1}$ must be larger than zero. Otherwise, we will have the problem of "division by zero" in Eqn. (3) if $K_{1}=0$, or negative value for cumulative interference $I$, which is unreasonable, if $K_{1}<0$ and $\alpha$ is an odd number. We next prove that, with our hexagon side length $h, K_{1}$ is always larger than zero.

According to the definition of $h$, we have that

$$
h=\min \left\{d_{1}, d_{2}\right\} \leq\left\{\begin{array}{l}
d_{1}, \\
d_{2} .
\end{array}\right.
$$

Then we have the following result

$\left\{\begin{array}{l}(1+\beta)^{3 h^{2}+3 h-1}<(1+\beta)^{3 d_{1}^{2}+3 d_{1}-1}=\sqrt{P_{M} /\left(N_{0} \beta\right)}, \\ (2 h)^{\alpha}<\left(2 d_{2}\right)^{\alpha}=\sqrt{P_{M} /\left(N_{0} \beta\right)} .\end{array}\right.$

It is clear that

$$
N_{0} \beta(1+\beta)^{3 h^{2}+3 h-1}(2 h)^{\alpha}<N_{0} \beta\left(\sqrt{P_{M} /\left(N_{0} \beta\right)}\right)^{2}<P_{M} .
$$

So we have

$$
\frac{1}{\beta(1+\beta)^{3 h^{2}+3 h-1}}-\frac{N_{0}(2 h)^{\alpha}}{P_{M}}>0 \Rightarrow K_{1}>0 .
$$

In conclusion, the value of $h$ validate the assignment of $K_{1}$.

\section{APPENDIX E \\ AN EXAMPLE OF AGgREgATION TREE CON- STRUCTION}

Fig. 12 illustrates the tree construction procedure with an example.

\section{APPENDIX F \\ Correctness OF LiNK Scheduling IN STEP S1}

We make use of the following theorem from [31] and Lemma 6 to complete our proof.

Theorem 7. (Groemer Inequality [31]) Suppose that $C$ is a compact convex set and $U$ is a set of points with mutual distances at least one. Then

$$
|U \cap C| \leq \frac{\operatorname{area}(C)}{\sqrt{3} / 2}+\frac{\operatorname{peri}(C)}{2}+1,
$$

where area $(C)$ and peri $(C)$ are the area and the perimeter of $C$, respectively.

Lemma 6. In a hexagon of side length $d$, there are at most $3 d^{2}+3 d+1$ nodes if the minimum distance between any two nodes is 1 .

Proof: Since hexagon is a compact convex set, on the basis of Theorem 9, we can have the maximum number of nodes in the hexagon as follows,

$$
|U \cap C| \leq \frac{6 \cdot 1 / 2 \cdot \sqrt{3} / 2 \cdot d^{2}}{\sqrt{3} / 2}+\frac{6 \cdot d}{2}+1=3 d^{2}+3 d+1 . \square
$$

We have that each pair of concurrent receivers in step S1 are separated by $2\left(K_{1}+1\right) h$ and $K_{1}=\left(6 X^{\prime}\right)^{\frac{1}{\alpha}}(1+$ $\left.\left(\frac{2}{\sqrt{3}}\right)^{\alpha} \frac{1}{\alpha-2}\right)^{\frac{1}{\alpha}}\left(\frac{1}{\beta(1+\beta)^{3 h^{2}+3 h-1}}-\frac{N_{0}(2 h)^{\alpha}}{P_{M}}\right)^{-\frac{1}{\alpha}}$ with $X^{\prime}=$ $3 h^{2}+3 h$.

Since the maximum number of signals that can be canceled at one receiver at a time is the maximum number of nodes in one hexagon minus 1 , we know, according to Lemma 6 , that at most $3 h^{2}+3 h=X^{\prime}$ signals can be canceled. Using the same approach with [27], i.e., assuming maximum power assignment at all concurrent transmitters when each pair of concurrent receivers are separated by a constant distance, the cumulative interference at any receiver with successive interference cancellation can be upperbounded as, 


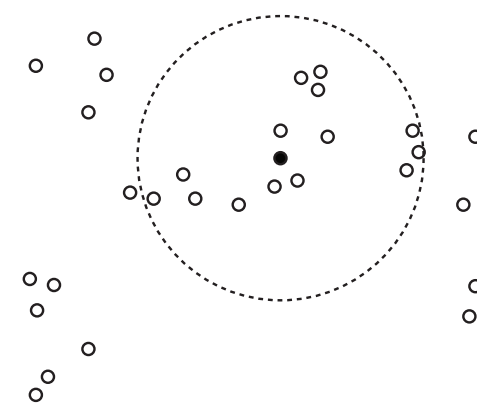

(a) Network topology with the dark node as the sink and the dashed circle as its maximum transmission range.

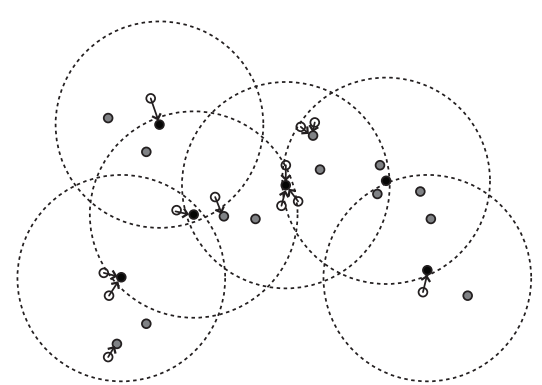

(d) Step T3.a with dark nodes as dominators, grey nodes as head dominatees and white nodes as non-head dominatees.

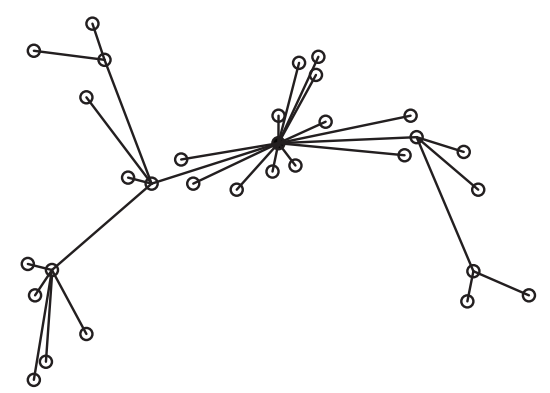

(b) BFS tree after step T1.
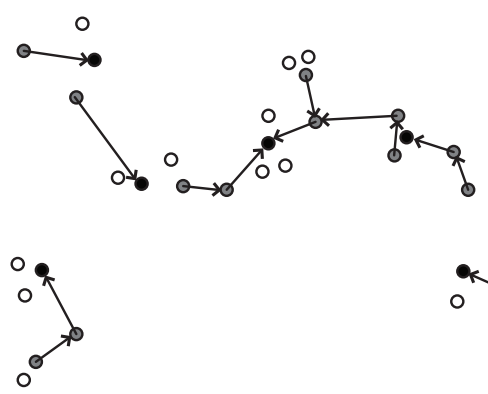

Step T3.b with dark nodes as dominators, grey nodes as head dominatees and white nodes as non-head dominatees.

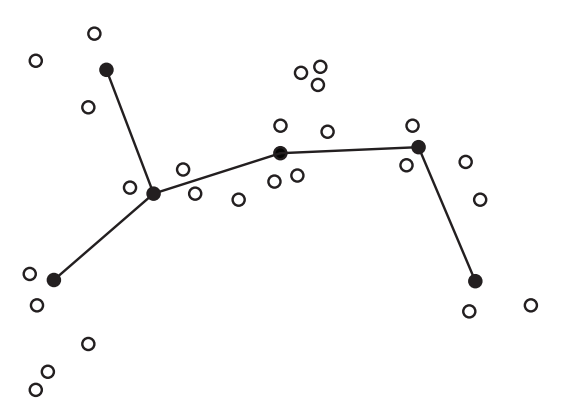

(c) CDS after step T2 with all dark nodes as the dominators.

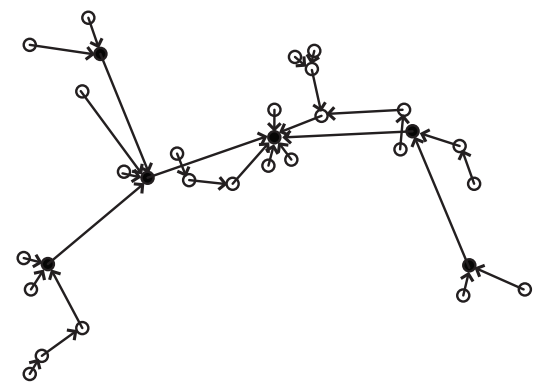

(f) Aggregation tree after step T3.

Fig. 12: An example of aggregation tree construction.

$$
\begin{aligned}
& X^{\prime}\left(6\left(\frac{1}{K_{1}}\right)^{\alpha}+\sum_{j=2}^{\infty} 6 j\left(\frac{2}{\sqrt{3} j K_{1}}\right)^{\alpha}\right) P_{M} /(2 h)^{\alpha} \\
= & 6 X^{\prime}\left(\frac{1}{K_{1}}\right)^{\alpha}\left(1+\sum_{j=2}^{\infty}\left(\frac{2}{\sqrt{3} j}\right)^{\alpha}\right) P_{M} /(2 h)^{\alpha} \\
= & 6 X^{\prime}\left(\frac{1}{K_{1}}\right)^{\alpha}\left(1+\left(\frac{2}{\sqrt{3}}\right)^{\alpha} \sum_{j=2}^{\infty} \frac{1}{j^{\alpha-1}}\right) P_{M} /(2 h)^{\alpha} \\
= & 6 X^{\prime}\left(\frac{1}{K_{1}}\right)^{\alpha}\left(1+\left(\frac{2}{\sqrt{3}}\right)^{\alpha} \frac{1}{\alpha-2}\right) P_{M} /(2 h)^{\alpha} \\
= & \frac{P_{M} /(2 h)^{\alpha}}{\beta(1+\beta)^{X^{\prime}-1}}-N_{0}=I .
\end{aligned}
$$

The power assignment is $P_{i}=\left(N_{0}+I\right) \beta(1+\beta)^{X^{\prime}-i} d_{i i}^{\alpha}$ for the $i^{t h}$ canceled link with length $d_{i i}$. Then we prove that the link can be correctly scheduled with successive interference cancellation with a valid SINR value,

$$
\begin{aligned}
& \frac{P_{i} / d_{i i}^{\alpha}}{N_{0}+I+\sum_{j=i+1}^{X^{\prime}} \frac{\left(N_{0}+I\right) \beta(1+\beta)^{X^{\prime}-j} d_{j i}^{\alpha}}{d_{j i}^{\alpha}}} \\
= & \frac{\left(N_{0}+I\right) \beta(1+\beta)^{X^{\prime}-i}}{N_{0}+I+\frac{\left(N_{0}+I\right) \beta\left((1+\beta)^{X^{\prime}-i}-1\right)}{\beta}}=\beta .
\end{aligned}
$$

We can conclude that each link transmission in step S1 is successful under the physical interference model with successive interference cancellation.

\section{APPENDIX G \\ Correctness OF LINK SCHEduling IN STEPS S2 AND S3}

We know that, in steps S2 and S3 of link scheduling, any two concurrent transmitters are separated by at least $\left(K_{2}+1\right) d_{M}$, where $K_{2}=\left(6 \beta\left(1+\left(\frac{2}{\sqrt{3}}\right)^{\alpha} \frac{1}{\alpha-2}\right)+1\right)^{1 / \alpha}$. For any scheduled link with length $r$, we have the power assignment $P=$
$N_{0} \beta\left(2-1 / K_{2}^{\alpha}\right) r^{\alpha}$. According to the conclusion in [27], the cumulative interference $I^{\prime}$ at any receiver is

$$
\begin{aligned}
I^{\prime} & \leq 6\left(\frac{1}{K_{2}}\right)^{\alpha}\left(1+\left(\frac{2}{\sqrt{3}}\right)^{\alpha} \frac{1}{\alpha-2}\right) \frac{P_{M}}{d_{M}^{\alpha}} \\
& =6 N_{0} \beta\left(\frac{1}{K_{2}}\right)^{\alpha}\left(1+\left(\frac{2}{\sqrt{3}}\right)^{\alpha} \frac{1}{\alpha-2}\right) \\
& =N_{0}\left(1-1 / K_{2}^{\alpha}\right) .
\end{aligned}
$$

So the SINR value for any scheduled link with length of $r$ should be

$$
\frac{P / r^{\alpha}}{N_{0}+I^{\prime}} \geq \frac{N_{0} \beta\left(2-1 / K_{2}^{\alpha}\right)}{N_{0}+N_{0}\left(1-1 / K_{2}^{\alpha}\right)}=\beta .
$$

We can conclude that each link transmission in steps S2 and S3 is successful under the physical interference model.

\section{APPEndix H Proof to Theorem 5}

The proof of Theorem 5 is based on the following lemma.

Lemma 3. A disk with radius $d$ can be covered by at most $\frac{2(d+h)(2 d-h)}{3 h^{2}}+1$ hexagons with side length $h$.

Proof: As illustrated by Fig. 13, we can divide the disk into 6 equal-sized non-overlapping cones, labeled $A$ to $F$. It is obvious that the maximum number of hexagons to cover the disk is at most 6 times the number to cover each cone.

In each cone, there are at most $\frac{1}{6}$ of a hexagon in the range of $\frac{1}{2} h$ to the center of the disk, $\frac{1}{6}+1$ hexagons in the range of $2 h, \frac{1}{6}+1+2$ hexagons in the range of $\frac{7}{2} h$, and so on. We can prove by induction that there are at most $1 / 6+\sum_{i=0}^{j} i$ hexagons in the range of $\frac{1+3 j}{2} h$ in each cone. Therefore, in a range of $d$, corresponding to $j=\left\lfloor\frac{2 d-h}{3 h}\right\rfloor$, there are at most $1 / 6+\frac{\frac{2 d-h}{3 h}\left(\frac{2 d-h}{3 h}+1\right)}{2}$ hexagons in one cone. We can then derive that there are at most $\frac{2(d+h)(2 d-h)}{3 h^{2}}+1$ hexagons in the entire disk with radius $d$. 


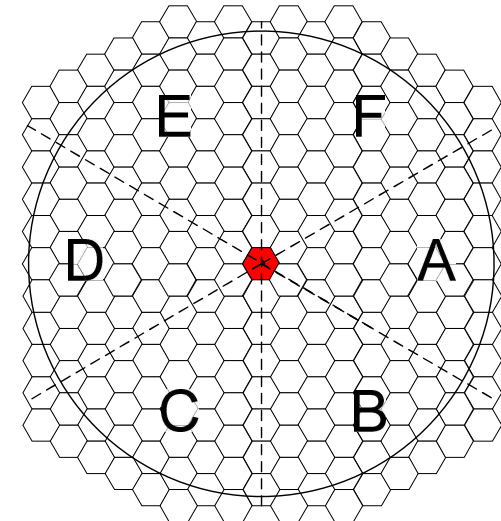

Fig. 13: An illustration of the proof of lemma 3.

Proof of Theorem 5: In step S1 of the link scheduling, each pair of concurrent receivers (head dominatees) are separated by at least a distance of $2\left(K_{1}+1\right) h$. So at least one head dominatee, in a disk of radius $2\left(K_{1}+1\right) h$, can successfully receive all the transmissions from the other dominatees in its hexagon in each time slot. In addition, there are at most $\frac{16}{3} K_{1}^{2}+12 K_{1}+7$ head dominatees in a disk of radius $2\left(K_{1}+1\right) h$ according to Lemma 3 . Therefore, the aggregation latency in this step is at most $\frac{16}{3} K_{1}^{2}+12 K_{1}+7$ time slots with at most $(1+X \tau)\left(\frac{16}{3} K_{1}^{2}+12 K_{1}+7\right)$ time units, which is a constant value.

When the first step finishes, there are at most $\frac{2\left(d_{M}+h\right)\left(2 d_{M}-h\right)}{3 h^{2}}+1$ remaining nodes in a disk of radius $d_{M}$, which have not transmitted.

In step S2 of the link scheduling in EMA-SIC, transmissions from head dominatees to their dominators along the respective minimum spanning trees are scheduled. Since each pair of concurrent transmitters are separated by a distance of at least $\left(K_{2}+1\right) d_{M}$, one head dominatee can be scheduled for transmission in a disk of radius $\left(K_{2}+1\right) d_{M}$ in each time slot. As at most $\frac{2\left(d_{M}+h\right)\left(2 d_{M}-h\right)}{3 h^{2}}+1$ head dominatees reside in a disk of radius $d_{M}$, there are at most $\left(K_{2}+1\right)^{2}\left(\frac{2\left(d_{M}+h\right)\left(2 d_{M}-h\right)}{3 h^{2}}+1\right)$ head dominatees to be scheduled in a disk of radius $\left(K_{2}+1\right) d_{M}$. Therefore, the aggregation latency in this step is at most $\left(K_{2}+\right.$ $1)^{2}\left(\frac{2\left(d_{M}+h\right)\left(2 d_{M}-h\right)}{3 h^{2}}+1\right)$ time units (SIC is not applied in Step S2), which is also a constant.

In step S3 of the link scheduling, transmissions from the dominators are scheduled along the tree connecting them to the sink. Since each dominator has a bounded degree in the connected dominant set [26], which is denoted by $c$ here, and at least one dominator can transmit in a disk of radius $\left(K_{2}+1\right) d_{M}$ in one time slot, each dominator needs to wait at most $\left(K_{2}+1\right)^{2} c$ time slots before it can transmit. In addition, the depth of the tree rooted at the sink spanning the connected dominating set is $O(D)$ [26]. Therefore, the aggregation latency in this step is at most $O\left(\left(K_{2}+1\right)^{2} c D\right)=O(D)$ time units (SIC is not utilized in Step S3).

In summary, the overall aggregation latency of EMASIC is upper-bounded by $O(D)$ time units. The aggregation latency approximation ratio, i.e., the ratio of this upper bound to the lower bound in Theorem 2, is upper-bounded by $O\left(D / \max \left\{D, \log _{X+1} n\right\}\right)=O(1)$.

\section{APPENDIX I \\ ProOF TO THEOREM 6}

We first present two lemmas and then the proof of Theorem 6.

Lemma 4. Let $V_{i}$ be the set of nodes in the disk of radius $d_{M}$ centered at a dominator $v_{i}$, including the dominator. Let $e_{1}, e_{2}, \ldots, e_{\left|V_{i}\right|-1}$ be the links in the minimum spanning tree of $V_{i}$ rooted at the dominator, derived using link weights proportional to $r^{\alpha}(\alpha \geq 2)$ for a link with length $r$. Then

$$
\sum_{k=1}^{\left|V_{i}\right|-1}\left|e_{k}\right|^{\alpha} \leq 6 d_{M}^{\alpha}
$$

Proof: Theorem 3 in [32] gives the following bound for the sum of squares of the link lengths in the minimum spanning tree which is constructed among the nodes in a unit disk centered at a dominator:

$$
\sum_{k=1}^{\left|V_{i}\right|-1}\left|e_{k}\right|^{2} \leq 6 .
$$

If we normalize the length of each link, $\left|e_{k}\right|$, to become $\frac{\left|e_{k}\right|}{d_{M}}$, our case will be equivalent to the case in [32]. Therefore, we have

$$
\sum_{k=1}^{\left|V_{i}\right|-1}\left(\frac{\left|e_{k}\right|}{d_{M}}\right)^{2} \leq 6 .
$$

As $\alpha \geq 2$ and $\left|e_{k}\right| \leq d_{M}$, we derive

$$
\sum_{k=1}^{\left|V_{i}\right|-1}\left(\frac{\left|e_{k}\right|}{d_{M}}\right)^{\alpha} \leq \sum_{k=1}^{\left|V_{i}\right|-1}\left(\frac{\left|e_{k}\right|}{d_{M}}\right)^{2} \leq 6 \Rightarrow \sum_{k=1}^{\left|V_{i}\right|-1}\left|e_{k}\right|^{\alpha} \leq 6 d_{M}^{\alpha} . \square
$$

Lemma 5. Suppose the minimum node degree of the nodes in the connected dominating set is $\delta$ and the sizes of the connected dominating set and maximum independent set with $P_{M}$ are $n_{c d s}$ and $n_{m i s}$, respectively. We have

$$
n_{c d s} \leq\left(1+\frac{1}{\delta-1}\right) n_{m i s} .
$$

Proof: According to the connected dominating set construction algorithm in [26], the dominators can be divided into two disjoint sets: $V_{I}$ and $V_{C}$, where $V_{I}$ is a set of independent nodes including the sink, $V_{C}$ is the set of nodes that are parents of the nodes in $V_{I}$ (expect the sink) in the breadth-first spanning tree, and $\left|V_{C}\right| \leq\left|V_{I}\right|-1$ [26]. We call the nodes in $V_{I}$ independent nodes and those in $V_{C}$ connector nodes. We next give a tighter relation between $\left|V_{C}\right|$ and $\left|V_{I}\right|$, which leads to this lemma.

Since the minimum node degree is $\delta$ in the connected dominating set, we know that one connector node at level $l+1$ can be the parent node of at least $\delta-1$ independent nodes in level $l+2$ and the child node of one independent node in level $l$. Suppose the number of the independent nodes in level $l+2$ is $\left|V_{I}(l+2)\right|$, then the number of the connector nodes in level $l+1$ is $\left|V_{C}(l+1)\right| \leq \frac{1}{\delta-1} \mid V_{I}(l+$ $2) \mid$. Suppose the total number of levels in the connected dominating set is $L$. For completeness, we have $\left|V_{I}(0)\right|=1$ and $\left|V_{I}(1)\right|=\left|V_{C}(0)\right|=\left|V_{C}(L-1)\right|=0$, since the sink, which is in $V_{I}$, is the only node at level 0 and there is no independent node in level 1 or connector node in level 0 or $L-1$. Then, we derive 


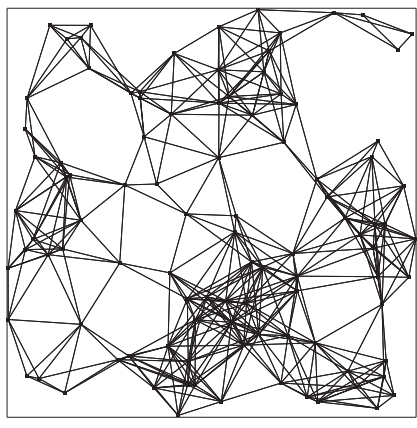

(a) Uniform

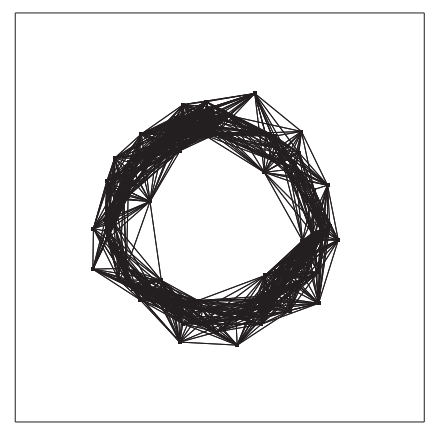

(b) Poisson

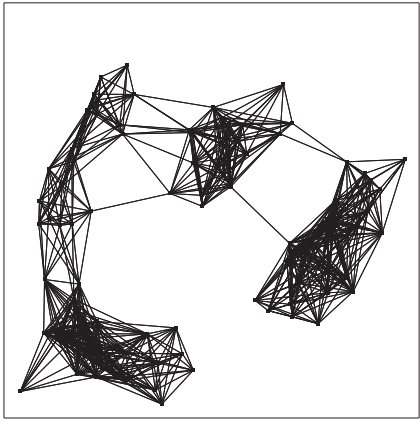

(c) Cluster

Fig. 14: Sample topologies of 100 nodes with different distributions.

$$
\begin{aligned}
\left|V_{C}\right|=\left|\bigcup_{l=0}^{L-1} V_{C}(l)\right|=\sum_{l=1}^{L-2}\left|V_{C}(l)\right| & \leq \frac{1}{\delta-1} \sum_{l=2}^{L-1}\left|V_{I}(l+1)\right| \\
& =\frac{1}{\delta-1}\left(\sum_{l=0}^{L-1}\left|V_{I}(l)\right|-1\right) \\
& =\frac{1}{\delta-1}\left(\left|V_{I}\right|-1\right) .
\end{aligned}
$$

Since $n_{c d s} \leq\left|V_{I}\right|+\left|V_{C}\right|$ and $\left|V_{I}\right| \leq n_{m i s}$, we have

$$
n_{c d s} \leq\left|V_{I}\right|+\left|V_{C}\right| \leq\left(1+\frac{1}{\delta-1}\right)\left|V_{I}\right| \leq\left(1+\frac{1}{\delta-1}\right) n_{m i s} . \square
$$

Proof of Theorem 6: In step S1 of the link scheduling with EMA-SIC, the length of the $i^{\text {th }}$ link in the interference cancellation sequence at each receiver (head dominator) satisfies $d_{i i} \leq 2 h$, and the transmission power assigned to the $i^{\text {th }}$ link to cancel satisfies $\left(N_{0}+I\right) \beta(1+\beta)^{X^{\prime}-i} d_{i i}^{\alpha} \leq$ $\left(N_{0}+I\right) \beta(1+\beta)^{X^{\prime}-i}(2 h)^{\alpha} \leq P_{M}$. The overall energy consumption to schedule the concurrent transmissions within one hexagon is

$$
\begin{aligned}
& \sum_{i=1}^{X^{\prime}}\left(N_{0}+I\right) \beta(1+\beta)^{X^{\prime}-i} d_{i i}^{\alpha} \\
\leq & \left(N_{0}+I\right) \beta(2 h)^{\alpha} \sum_{i=1}^{X^{\prime}}(1+\beta)^{X^{\prime}-i} \\
= & \frac{1+\beta}{\beta}\left(N_{0}+I\right) \beta(1+\beta)^{X^{\prime}-1}(2 h)^{\alpha}-\left(N_{0}+I\right)(2 h)^{\alpha} \\
< & \frac{1+\beta}{\beta} P_{M} .
\end{aligned}
$$

Therefore, the overall energy consumption to aggregate data within one hexagon, from all the non-head dominatees to the head dominatees, is upper-bounded by $\frac{1+\beta}{\beta} P_{M}$.

Consider a maximum independent set with $P_{M}$ of the given network with size $n_{m i s}$. Since a maximum independent set with $P_{M}$ is also a dominating set of the network, the disks with radius $d_{M}$ centered at each node in the maximum independent set with $P_{M}$ can cover the network. From Lemma 3, we know that there are at most $C=\frac{2\left(d_{M}+h\right)\left(2 d_{M}-h\right)}{3 h^{2}}+1$ hexagons of side length $h$ in each disk with radius $d_{M}$. Therefore, there are at most $C n_{m i s}$ hexagons in the entire network. The overall energy consumption for transmissions in all the hexagons in step $\mathrm{S} 1$ is then at most $C n_{m i s} \frac{1+\beta}{\beta} P_{M}$.

We next analyze the energy consumption in step S2 and step S3 of the link scheduling in EMA-SIC.

Suppose the size of the maximum independent set with $P_{M}$ in the network is $n_{m i s}$ and the size of the constructed connected dominating set is $n_{c d s}$. Let $V_{i}$ be the set of head dominatee nodes of any given dominator $v_{i}$, and $e_{1}, e_{2}, \ldots, e_{\left|V_{i}\right|}$ be the links in the minimum spanning tree connecting $V_{i}$ to $v_{i}$. Since the energy consumption on each link $e_{k}$ is $N_{0} \beta\left(2-1 / K_{2}^{\alpha}\right)\left|e_{k}\right|^{\alpha}$, we derive the upper bound on overall energy consumption to transmit data from all the head dominatees to the dominator as follows (the inequality is based on Lemma 4)

$$
\begin{aligned}
\sum_{k=1}^{\left|V_{i}\right|} N_{0} \beta\left(2-1 / K_{2}^{\alpha}\right)\left|e_{k}\right|^{\alpha} & =N_{0} \beta\left(2-1 / K_{2}^{\alpha}\right) \sum_{k=1}^{\left|V_{i}\right|}\left|e_{k}\right|^{\alpha} \\
& \leq 6 N_{0} \beta\left(2-1 / K_{2}^{\alpha}\right) d_{M}^{\alpha} .
\end{aligned}
$$

Lemma 5 shows that there are at most $\left(1+\frac{1}{\delta-1}\right) n_{\text {mis }}$ dominators in the connected dominating set. So energy consumption of at most $\left(1+\frac{1}{\delta-1}\right) n_{m i s} \times 6 N_{0} \beta\left(2-1 / K_{2}^{\alpha}\right) d_{M}^{\alpha}$ is needed to aggregate data from all dominatees in the network to the connected dominating set, and energy consumption of at most $\left(1+\frac{1}{\delta-1}\right) n_{m i s} \times N_{0} \beta\left(2-1 / K_{2}^{\alpha}\right) d_{M}^{\alpha}$ is needed to aggregate data from all the dominators to the sink. Therefore, the overall energy consumption with EMA-SIC in steps $\mathrm{S} 2$ and $\mathrm{S} 3$ of the link scheduling is at most $7 N_{0} \beta\left(1+\frac{1}{\delta-1}\right) n_{m i s}\left(2-1 / K_{2}^{\alpha}\right) d_{M}^{\alpha}$.

Dividing the sum of the upper bounds on energy consumption in all three steps by the lower bound in Theorem 1 , we have an energy consumption approximation ratio of at most

$$
\begin{aligned}
& \frac{C n_{m i s} \frac{1+\beta}{\beta} P_{M}+7 N_{0} \beta\left(1+\frac{1}{\delta-1}\right) n_{m i s}\left(2-1 / K_{2}^{\alpha}\right) d_{M}^{\alpha}}{N_{0} \beta \frac{\left(n_{m i s} d_{M}\right)^{\alpha}}{n^{\alpha-1}}} \\
= & \left(\frac{C(1+\beta)}{\beta}+7\left(1+\frac{1}{\delta-1}\right)\left(2-1 / K_{2}^{\alpha}\right)\right)\left(\frac{n}{n_{m i s}}\right)^{\alpha-1},
\end{aligned}
$$

where $N_{0} \beta d_{M}^{\alpha}=P_{M}$ is used.

Since $\Delta \geq n / n_{m i s}$ while $C, \beta, \delta, K_{2}$ and $\alpha$ are constants, we further derive that the energy consumption approximation ratio is upper-bounded by

$\left(\frac{C(1+\beta)}{\beta}+7\left(1+\frac{1}{\delta-1}\right)\left(2-1 / K_{2}^{\alpha}\right)\right) \Delta^{\alpha-1}=O\left(\Delta^{\alpha-1}\right) . \square$

\section{APPENDIX J}

Fig. 14 in Appendix $\mathrm{J}$ gives an illustration of network topologies with 100 nodes under different distributions.

\section{APPENDIX K \\ ADDITIONAL SIMULATION RESULTS}

This section includes additional simulation results, under cases where $\mathrm{Li}$ et al.'s algorithm exerts its effectiveness. 
Complete simulation results, with various distribution patterns, network scales, node numbers and $(\alpha, \beta)$ combinations, have 1080 figures and are online available in our technical report [29].

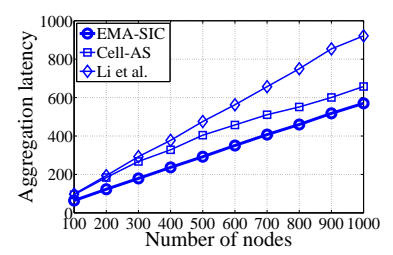

(a) $\alpha=4, \beta=2$

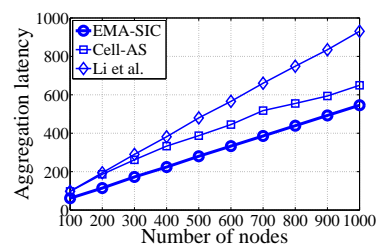

(c) $\alpha=5, \beta=4$

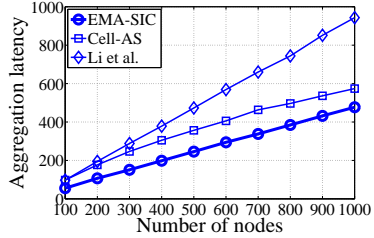

(b) $\alpha=5, \beta=2$

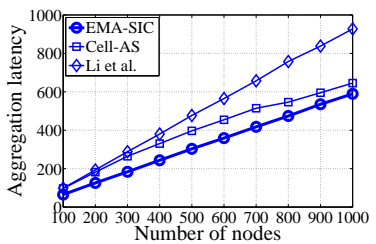

(d) $\alpha=5, \beta=6$
Fig. 15: Aggregation latency (time units) comparison under selected network settings in a $180 \times 180 \mathrm{~m}^{2}$ area.

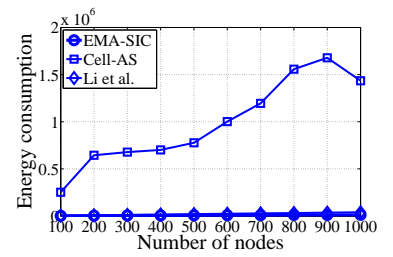

(a) $\alpha=4, \beta=2$

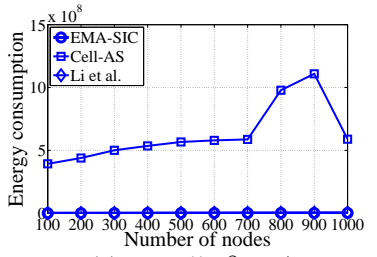

(c) $\alpha=5, \beta=4$

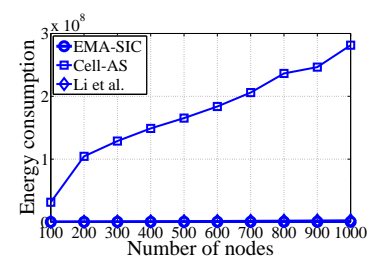

(b) $\alpha=5, \beta=2$

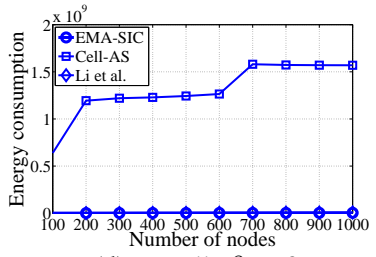

(d) $\alpha=5, \beta=6$
Fig. 16: Energy consumption (joule) comparison under selected network settings in a $180 \times 180 \mathrm{~m}^{2}$ area.

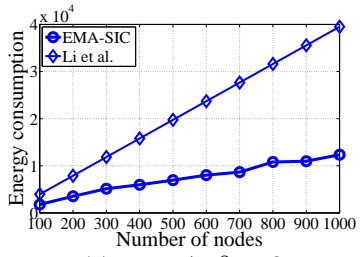

(a) $\alpha=4, \beta=2$

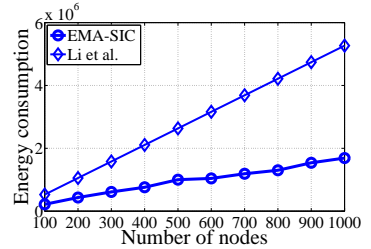

(c) $\alpha=5, \beta=4$

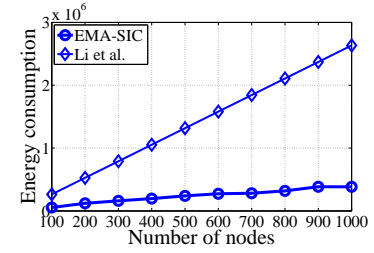

(b) $\alpha=5, \beta=2$

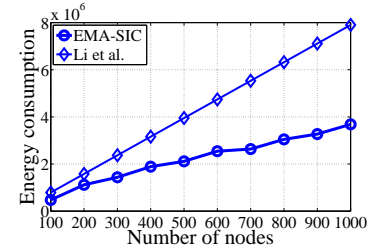

(d) $\alpha=5, \beta=6$
Fig. 17: A separate comparison of energy consumption (joule) between EMA-SIC and Li et al.'s algorithm under selected network settings in a $180 \times 180 \mathrm{~m}^{2}$ area.

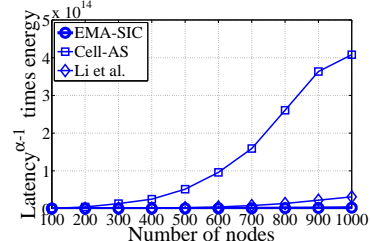

(a) $\alpha=4, \beta=2$

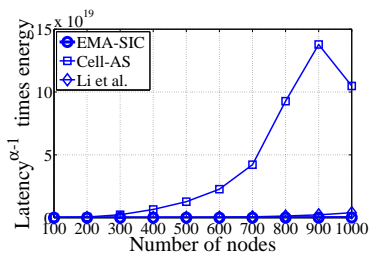

(c) $\alpha=5, \beta=4$

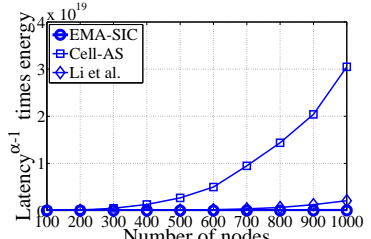

(b) $\alpha=5, \beta=2$

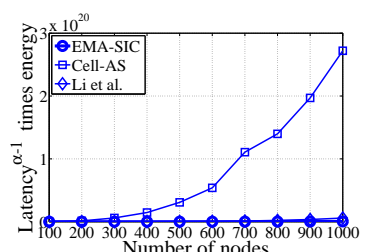

(d) $\alpha=5, \beta=6$
Fig. 18: Latency-energy tradeoff comparison under selected network settings in a $180 \times 180 \mathrm{~m}^{2}$ area.

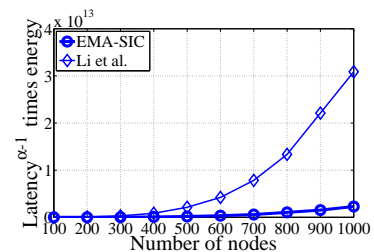

(a) $\alpha=4, \beta=2$

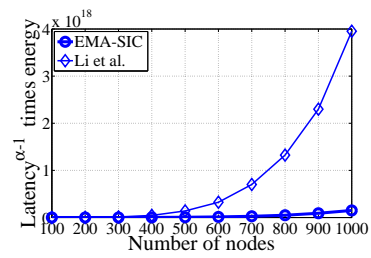

(c) $\alpha=5, \beta=4$

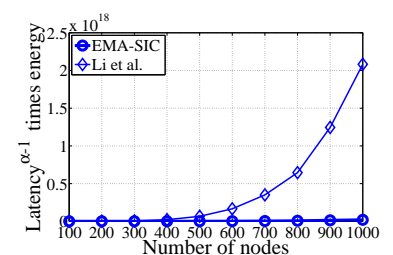

(b) $\alpha=5, \beta=2$

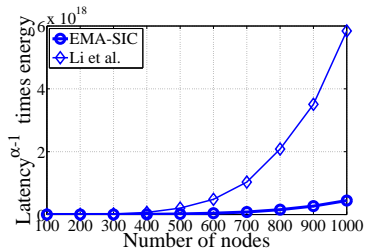

(d) $\alpha=5, \beta=6$
Fig. 19: A separate comparison of latency-energy between EMA-SIC and $\mathrm{Li}$ et al.'s algorithm under selected network settings in a $180 \times 180 \mathrm{~m}^{2}$ area.

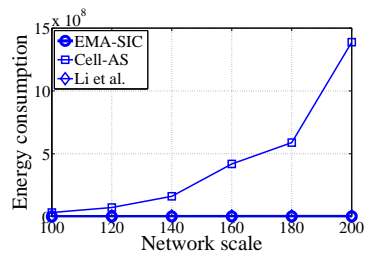

(a) $\alpha=5, \beta=4,3$ algorithms

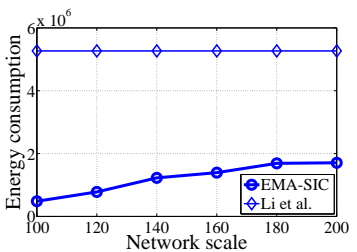

(c) $\alpha=5, \beta=4,2$ algorithms

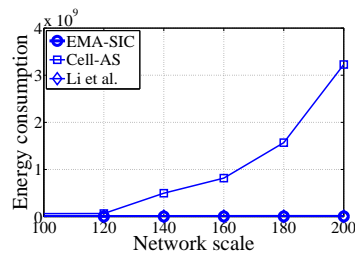

(b) $\alpha=5, \beta=6,3$ algorithms

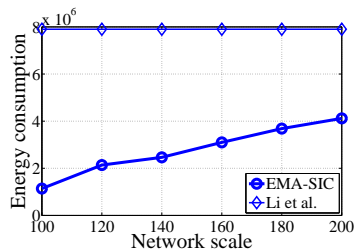

(d) $\alpha=5, \beta=6,2$ algorithms
Fig. 20: Impact of network scale on the energy consumption (joule) under selected network settings with uniform distribution and 1000 nodes. 


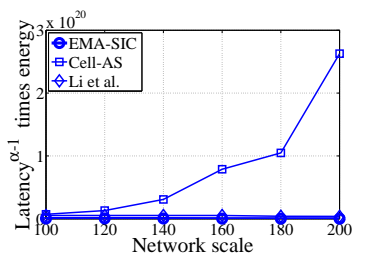

(a) $\alpha=5, \beta=4,3$ algorithms

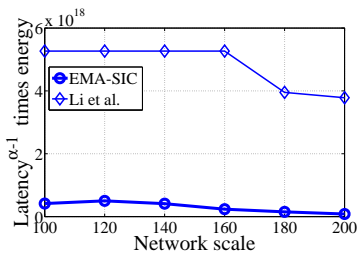

(c) $\alpha=5, \beta=4,2$ algorithms

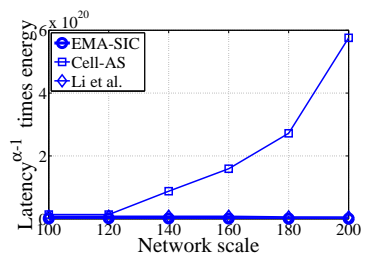

(b) $\alpha=5, \beta=6,3$ algorithms

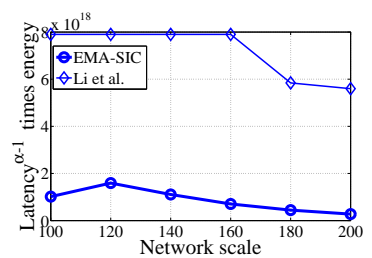

(d) $\alpha=5, \beta=6,2$ algorithms

Fig. 21: Impact of network scale on the latency-energy tradeoff under selected network settings with uniform distribution and 1000 nodes. 